\title{
Multiuser Communication through Power Talk in DC MicroGrids
}

\author{
Marko Angjelichinoski ${ }^{\dagger}$, Student Member, IEEE, \\ Čedomir Stefanović ${ }^{\dagger}$, Member, IEEE, Petar Popovski ${ }^{\dagger}$, Senior Member, IEEE, \\ Hongpeng Liu ${ }^{\ddagger}$, Member, IEEE, Poh Chiang Loh*, \\ Frede Blaabjerg*, Fellow, IEEE
}

\begin{abstract}
Power talk is a novel concept for communication among control units in MicroGrids (MGs), carried out without a dedicated modem, but by using power electronics that interface the common bus. The information is transmitted by modulating the parameters of the primary control, incurring subtle power deviations that can be detected by other units. In this paper, we develop power talk communication strategies for DC MG systems with arbitrary number of control units that carry out all-to-all communication. We investigate two multiple access strategies: 1) TDMA, where only one unit transmits at a time, and 2) full duplex, where all units transmit and receive simultaneously. We introduce the notions of signaling space, where the power talk symbol constellations are constructed, and detection space, where the demodulation of the symbols is performed. The proposed communication technique is challenged by the random changes of the bus parameters due to load variations in the system. To this end, we employ a solution based on training sequences, which re-establishes the signaling and detection spaces and thus enables reliable information exchange. The presented results show that power talk is an effective solution for reliable communication among units in DC MG systems.
\end{abstract}

\section{Index Terms}

\footnotetext{
${ }^{\dagger}$ The authors are with the department of Electronic systems, Aalborg University, Denmark (e-mail: $\{$ maa,cs,petarp\}@es.aau.dk)

*The authors are with the department of Energy technology, Aalborg University, Denmark (e-mail: $\{$ pcl,fbl $\} @ e t . a a u . d k)$.

${ }^{\ddagger}$ The author is with the department of Electrical Engineering, Harbin Institute of Technology, Harbin, China (e-mail: hpl@et.aau.dk).
}

The work presented in this paper was supported in part by EU, under grant agreement no. 607774 "ADVANTAGE". 
Power Talk, MicroGrid Communications, Droop Control, Signaling Space, Detection Space

\section{INTRODUCTION}

MicroGrids (MGs) are localized clusters of Distributed Energy Resources (DERs) and loads interfacing one or multiple buses via flexible power electronic interfaces, able to operate both in connected (to the main grid) or standalone mode [1]. The future power grid is envisioned as interconnected mesh of MGs, enabling flexible operation and improving the efficiency [2], [3]. Variety of practical MG applications have emerged, differing significantly in scope, size and in the demands for communication and signal processing support. The MG operation, especially in standalone mode [4], relies on advanced control mechanisms assisted by communication technologies. MG control is commonly based on a three-level hierarchy: primary, secondary and tertiary [5]-[7]. The primary level provides fast control of the basic MG operation, such as bus voltage and/or frequency control based on predefined references. The secondary and tertiary levels provide slower control mechanisms for enhancing the power quality, by setting the references for the primary level and optimizing the MG operation in grid-connected mode.

The traditional design of primary control avoids use of communications, due to the fact that most of the existing communication standards (particularly wireless ones), are not designed to support the machine-type control traffic in power grid applications, i.e., are unable to guarantee high reliability and constant availability. Hence, the primary control is traditionally designed in a distributed manner, where each unit uses only locally available measurements [5]-[7]. On the other hand, recent works consider the use of the existing MG power equipment, i.e., power electronics and power lines, as a means to exchange control messages [8]-[11]. An obvious solution in this respect is to use power line communications (PLC) [12]. However, for the emerging MG applications, where the focus is on small, isolated and localized systems operating in standalone mode, PLC might prove to be a cost-inefficient and overly complex solution.

This paper presents a novel, inexpensive, reliable and low-bandwidth communication solution, designed specifically for MGs operating in standalone mode and implemented using only the existing power equipment. We refer to it as power talk, as it modulates information over the main bus of the MG through subtle deviations of the power supplied by each unit. In particular, power talk exploits the flexibility of the electronic inverters, which modulate the parameters of the primary loops that control the parameters of the common bus and in this way exchange 
information among the MG units. The availability/reliability of power talk matches the ones of the MG bus, circumvents the use of additional hardware and requires only software enhancements.

Power talk has been introduced in [13], [14], through a simplistic DC MG system with two units in a one-way communication scenario. The focus of [13] is on enabling reliable communication without precise knowledge of the system configuration and of the load; it is shown that using a special input symbol in a role of a pilot transforms the MG bus into some of the well-studied channels. [14] represents the unknown system configuration and load variations through a Thevenin equivalent, whose parameters determine the channel state that can be estimated. This enables to design power talk constellations of an arbitrary order that perform optimally in terms of symbol error probability.

In this paper, we extend the power talk to scenarios with multiple units, in which each unit communicates with all other units in the system. The contributions can be summarized as follows:

- We develop and analyze power talk strategies both for Time Division Multiple Access (TDMA), where a single unit transmits at a time, and Full Duplex (FD), where all units transmit and receive simultaneously.

- We present the concepts of signaling and detection spaces, based on which we develop communication strategies. In particular, we design the signaling space by taking into account the control parameters that do not violate operational constraints of the MG. We then investigate the detection space, i.e., the local set of voltages and currents that a unit can observe, based on which the demodulation is implemented.

- We investigate simple protocol designs that deal with the problems of unknown system configuration and variable loads. These protocols rely on using training sequences that reset the detection space at each receiver and foster reliable communications over the MG.

The rest of the paper is organized as follows. Section $\amalg$ introduces the core ideas and implementation of power talk in DC MGs. Section III illustrates the communication principles of power talk through two simple, but insightful examples. Section IV presents the general communication model of power talk in DC MG. Section $\mathrm{V}$ investigates signaling and detection spaces and shows how to modulate and demodulate power talk symbols under MG operating constraints. Section VI addresses design aspects of fully operational communication protocols based on power talk. Section VII presents the performance evaluation. Finally, Section VIII concludes the paper. 


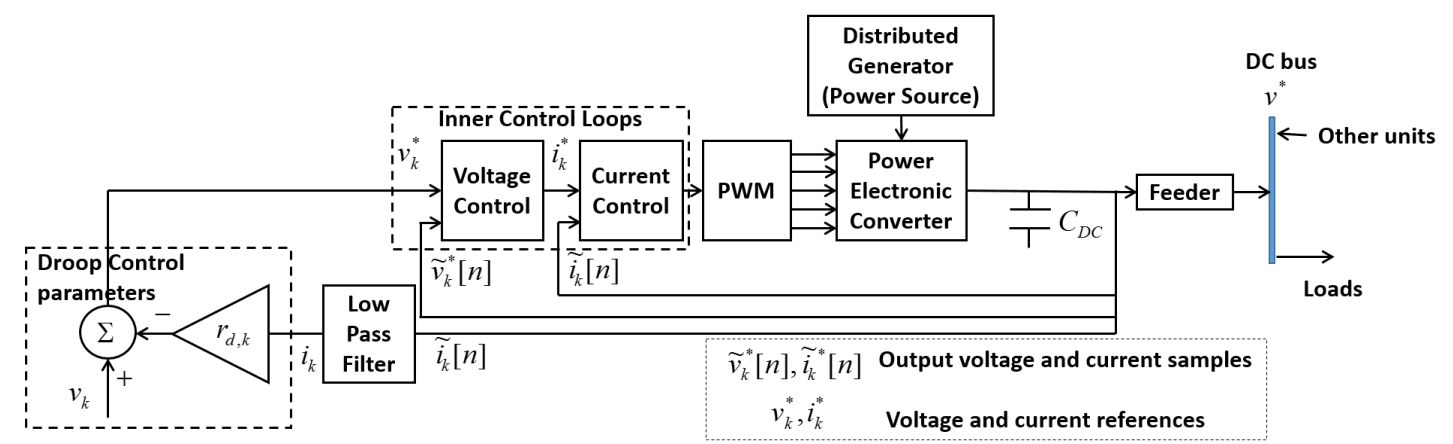

(a) Primary control loops of VSC $k$ in standalone operation.

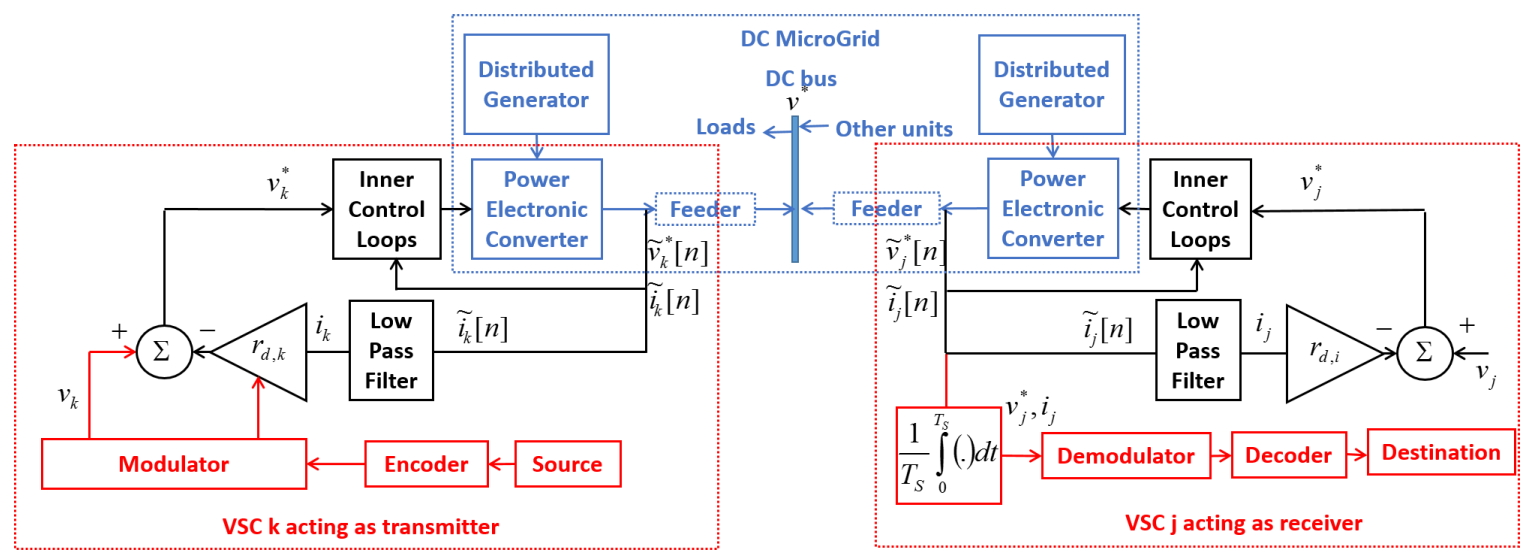

(b) DC MG as communication system.

Fig. 1. Overlaying communications over MG control.

\section{COMmunication through Microgrid CONTROL}

Fig. [1[a) shows the primary control diagram of a unit $k$ that operates as a Voltage Source Converter (VSC) and participates in the voltage regulation in the MG.1 The unit interfaces the common bus through power electronics that implements the inner current and voltage control loops. These loops are usually very fast (of the order of $\mathrm{kHz}$ ), enforcing the output current $i_{k}$ and voltage $v_{k}^{*}$ to follow the predefined references, as shown on Fig. 11[a),

The bus voltage reference $v_{k}^{*}$ that is fed to the inner voltage control loop of the unit $k$ is set according to the droop law [15]-[18]:

$$
v_{k}^{*}=v_{k}-r_{d, k} i_{k}
$$

${ }^{1} \mathrm{~A}$ unit can also operate as a Current Source Converter (CSC), when it does not use the inner voltage loop and does not participate in the voltage regulation. Also, a unit can, in principle, switch between the VSC and CSC modes seamlessly; we consider only VSC units in the paper, as their task is to control the bus voltage and power sharing in the standalone mode. 
where $v_{k}$ and $r_{d, k}$ are the droop parameters, namely the reference voltage and the virtual resistance (also referred to as droop slope), and $i_{k}$ is the output current. Parameters $v_{k}$ and $r_{d, k}$ are controllable; in standalone mode their values are usually set to enable proportional power sharing based on the ratings of the units. The basic idea of power talk is to change $v_{k}$ and $r_{d, k}$ in a controlled manner, thereby inducing variations in the output voltage $v_{k}^{*}$ and current $i_{k}$ that can be detected by other units in the system, thus leading to information exchange, see Fig. 11(b),

In the following, we list the assumptions used throughout the manuscript. Our focus is on small DC MGs, where all units are connected to a single common bus through feeder lines with negligible resistances. This assumption is valid for localized, isolated systems, expected to operate frequently in standalone mode [9], [10], [18]. For simplicity, we assume that VSCs in the MG supply a collection of loads aggregated in a single, purely resistive load, denoted by $R$, whose instantaneous value is denoted by $r$. We note that same concepts can be used with minor modifications for mixture of loads, including constant power load. We consider all-to-all communication scenario in which the time is slotted, where VSC units in the system maintain slot synchronization. The slot duration $T_{s}$ complies with the control bandwidth of the inner control loops and allows the system to reach steady-state [16]. In practice $T_{s}$ should be of the order of milliseconds. Each VSC samples the voltage and the output current with frequency $f_{o}$ of the order of $\mathrm{kHz}$ [17]. Power talk uses the averages of $f_{o} T_{s}$ samples during a single slot, i.e., all voltages and currents are averaged over slot duration $T_{s}$ with sampling frequency $f_{o}$, see Fig. 1(b). Finally, we consider only binary power talk and note that the developed techniques can be straightforwardly generalized to higher order modulations.

Under the above assumptions, for general DC MG system with $K$ VSCs and each of them using (11) to regulate output voltage and current, the common bus voltage $v^{*}$ in steady state is:

$$
v^{*}=\frac{\sum_{k=1}^{K} \frac{v_{k}}{r_{d, k}+r_{l, k}}}{\frac{1}{r}+\sum_{k=1}^{K} \frac{1}{r_{d, k}+r_{l, k}}} \approx \frac{\sum_{k=1}^{K} \frac{v_{k}}{r_{d, k}}}{\frac{1}{r}+\sum_{k=1}^{K} \frac{1}{r_{d, k}}},
$$

where the approximation holds for negligible resistances of the feeder lines, $r_{l, 1} \approx \ldots \approx r_{l, K} \approx 0$. The output current from VSC $k$ in steady state is:

$$
i_{k}=\frac{v_{k}-v^{*}}{r_{d, k}}
$$

i.e., the output currents $i_{k}$ from each VSC are determined by the bus voltage. Therefore, the bus voltage $v^{*}$ is the only degree of freedom that could be used for power talk in DC MGs. 


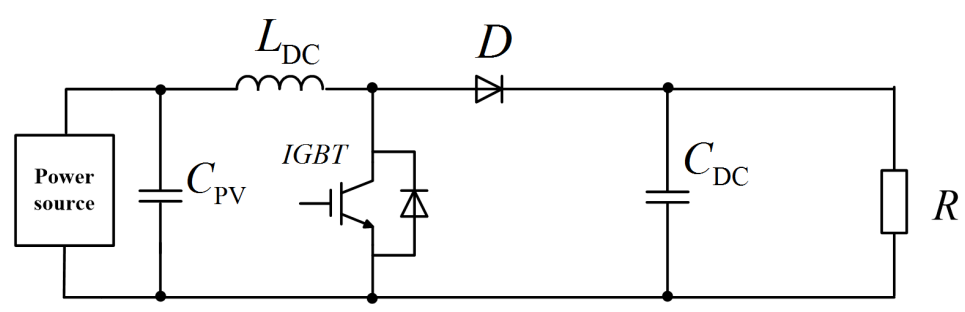

Fig. 2. DC-DC boost converter topology of a VSC unit.

TABLE I

DC MG PARAMETERS SIMULATED WITH PLECS.

\begin{tabular}{|c||c|c|c|c|c|c|c|c|c|c|}
\hline Parameter & $T_{s}$ & $f_{o}$ & $L_{D C}$ & $C_{P V}=C_{D C}$ & $V_{\max }$ & $V_{\min }$ & $I_{k, \max }$ & $\left(v_{k}^{\mathrm{n}}, r_{d, k}^{\mathrm{n}}\right)$ & $R_{\min }$ & $R_{\max }$ \\
\hline Value & $10 \mathrm{~ms}$ & $10 \mathrm{kHz}$ & $5 \mathrm{mH}$ & $470 \mu \mathrm{F}$ & $400 \mathrm{~V}$ & $390 \mathrm{~V}$ & $5 \mathrm{~A}$ & $(400 \mathrm{~V}, 2 \Omega)$ & $50 \Omega$ & $250 \Omega$ \\
\hline
\end{tabular}

Alternatively, this can be represented through a single parameter that is the output power $P_{k}=$ $v^{*} i_{k}$ that VSC $k$ is supplying to the bus, leading to the term power talk.

Finally, we note that all concepts, techniques and results presented in the paper were verified using PLECS $₫$ (Piecewise Linear Electrical Circuit Simulation) simulator integrated with

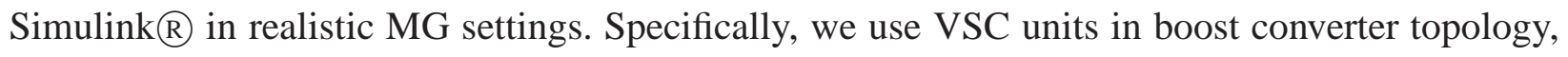
see Fig. 2 interfacing DER to the main bus. IGBT represents the insulated-gate bipolar transistor with switching frequency $f_{o}, D$ is a fast recovery diode, $L_{D C}$ is the boost inductor and $C_{D C}$ is the output capacitor. We simulate a low-voltage DC MG with allowable voltage deviation on the main bus $V_{\min } \leq v^{*} \leq V_{\max }$. The maximum current rating of VSC $k$, related to the maximum output power of the respective DG is denoted by $I_{k, \max }$. In nominal mode, VSC $k$ operates with droop parameters $v_{k}^{\mathrm{n}}$ and $r_{d, k}^{\mathrm{n}}$, designed to enable proportional power sharing based on the rating of the unit, satisfying $r_{d, k}^{\mathrm{n}}=\frac{v_{k}^{\mathrm{n}}-V_{\min }}{I_{k, \max }}$. The system is dimensioned to supply a collection of loads, equivalently represented with a single resistor $R$ whose instantaneous value varies in the range $r \in\left[R_{\min }, R_{\max }\right]$. The values of the parameters are summarized in Table I. The slot duration $T_{s}$ depends on the control bandwidth of the system. The investigations performed using the simulated topology showed that for $T_{s} \geq 1 \mathrm{~ms}$, the system reaches a steady state. Unless otherwise stated, in the rest of the text we use slot duration of $T_{s}=10 \mathrm{~ms}$. 


\section{Two ILlustrative EXAMPLES}

In this section, we present two toy examples of DC MGs, which capture the essence of power-talk concepts in all-to-all communication setup.

A. DC MG with two units: TDMA and FD communication strategies

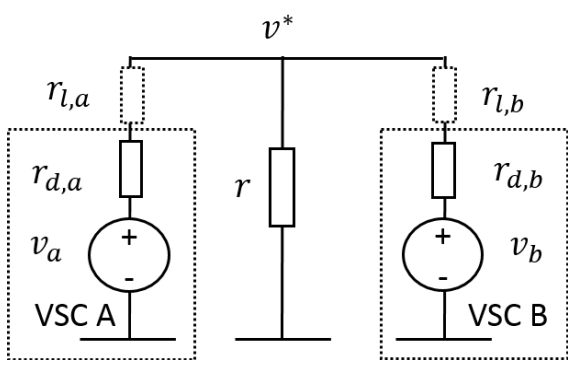

Fig. 3. DC MG system with two VSC units in steady-state.

Fig. 3 illustrates a two unit DC MG. The VSCs maintain the common bus voltage $v^{*}$ through droop control and supply load $r$. The droop control parameters of each VSC are denoted by $v_{a}$, $r_{d, a}$ and $v_{b}, r_{d, b}$. As already noted, the feeder-line resistances are neglected, i.e., $r_{l, a} \approx r_{l, b} \approx 0$. Each VSC locally observes the bus voltage $v^{*}$ and output current $i_{k}, k \in\{a, b\}$. Based on these observations, VSC $k$ constructs its local $v-i$ diagram as a the set of the outputs $\left(v^{*}, i_{k}\right)$, where each point in the diagram represents power $P_{k}=v^{*} i_{k}$ that VSC is supplying to the system.

The output power $P_{k}$ depends on: (i) the (own) droop parameters of VSC $k$, (ii) the droop parameters of the other VSC, and (iii) the value of the load $r$. Assume that $r$ does not change during the slot. Then, by measuring its own supplied power $P_{k}$, VSC $k$ can infer the droop parameters of the other unit. Specifically, by tracking the point $\left(v^{*}, i_{k}\right)$ in the local $v-i$ diagram, each VSC determines how much power $P_{k}$ it is providing and implicitly learns the droop parameters of the other VSC. This is the basic, underlying principle of power talk: each unit $k$ transmits information by changing its local droop control variables $v_{k}$ and $r_{d, k}$ and receives information by observing the local power output $P_{k}$ and detecting the corresponding point $\left(v^{*}, i_{k}\right)$ in the local $v-i$ diagram. Henceforth, we refer to the $v-i$ diagram as the detection space.

Denote the droop parameters of VSC $k$ as the input $\mathbf{x}_{k}$ :

$$
\mathbf{x}_{k}=\left(v_{k}, r_{d, k}\right), k \in\{a, b\}
$$


In nominal mode of operation, when not "power talking", the droop parameters have values $\mathbf{x}_{k}^{\mathrm{n}}=$ $\left(v_{k}^{\mathrm{n}}, r_{d, k}^{\mathrm{n}}\right), k \in\{a, b\}$, see Section 【. When transmitting, VSC $k$ uses two different combinations to represent the value of the transmitted bit $b_{k}$ :

$$
\begin{aligned}
& \mathbf{x}_{k}^{0}=\left(v_{k}^{0}, r_{d, k}^{0}\right) \leftrightarrow " 0 ", \\
& \mathbf{x}_{k}^{1}=\left(v_{k}^{1}, r_{d, k}^{1}\right) \leftrightarrow " 1 "
\end{aligned}
$$

and we refer to combination $\mathbf{x}_{k}^{b_{k}}$ as an input symbol. In the rest of the paper, we focus on the case where all units use the same symbols for signaling:

$$
\mathbf{x}_{k}^{0} \equiv \mathbf{x}^{0}, \mathbf{x}_{k}^{1} \equiv \mathbf{x}^{1}, k \in\{a, b\}
$$

This is the simplest case to deal with and provides valuable guidelines for designing power talk protocols. In principle, $\mathrm{x}^{1}$ and $\mathrm{x}^{0}$ can be chosen arbitrarily, as long as they comply to the operational constraints, as elaborated in Section $\mathrm{V}$

We denote the locally observed voltage and current at VSC $k$, i.e., the output symbol, by:

$$
\mathbf{s}_{k}=\left(v^{*}, i_{k}\right), k \in\{a, b\} .
$$

Each value of $\mathbf{s}_{k}$ in the detection space is associated to output power $P_{k}=v^{*} i_{k}$ and depends on the values of droop parameters of both units. In further text, we characterize $\mathbf{s}_{k}$ in the case of Time Division Multiple Access (TDMA) and Full Duplex (FD) approaches to power talk.

TDMA: Here we assume that the scheduling of the units is done in some predetermined manner, such that in each time slot only one active unit sends information over the MG, while the rest of the units operate in the nominal mode. Assuming that $r$ does not change, it can be seen that the value of the output symbol at all units depends only on the value of the input symbol sent by the active unit VSC $k$ :

$$
\mathbf{s}_{j}=\mathbf{s}_{j}\left(\mathbf{x}^{b_{k}}\right)=\mathbf{s}_{j}\left(b_{k}\right) \text { and } P_{j}=P_{j}\left(\mathbf{x}^{b_{k}}\right)=P_{j}\left(b_{k}\right), j \in\{a, b\}
$$

where $b_{k}$ is the information bit transmitted by VSC $k$. Without loss of generality, we assume that, when VSC $k$ is active, the inputs $\mathrm{x}^{1}$ and $\mathrm{x}^{0}$ satisfy:

$$
P_{k}(1)>P_{k}^{\mathrm{n}}>P_{k}(0), k \in\{a, b\},
$$

where $P_{k}^{\mathrm{n}}$ is the output power of VSC $k$ when all VSCs operate in the nominal mode. This implies that, if the load $r$ is stable, the output power of the receiving VSC $j$ satisfies:

$$
P_{j}(1)<P_{j}^{\mathrm{n}}<P_{j}(0), j \neq k \text {. }
$$




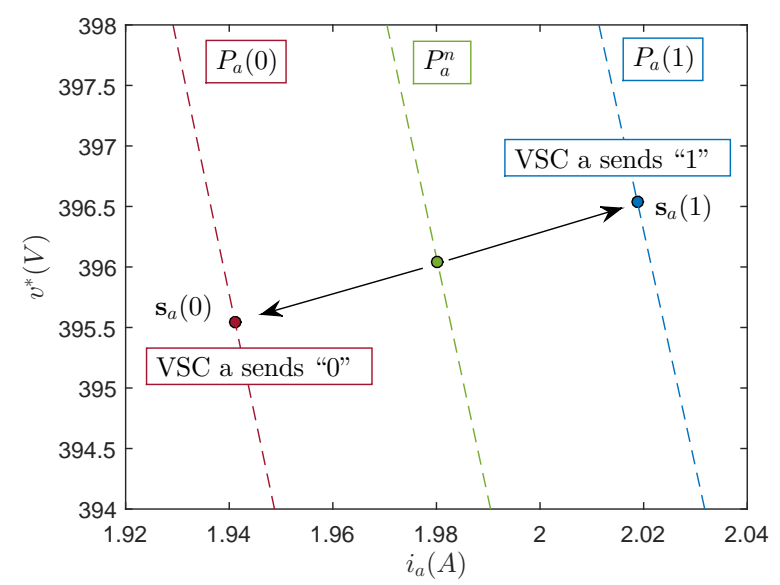

(a) VSC $a$ (the transmitter).

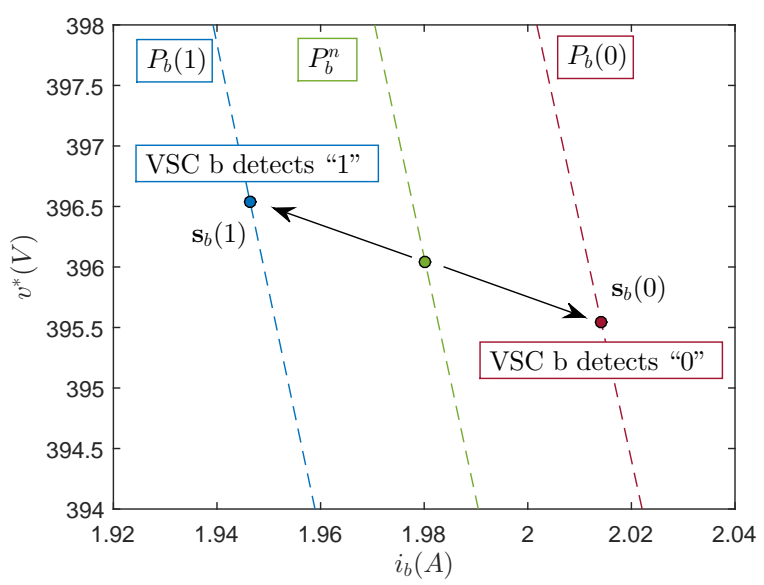

(b) VSC $b$ (the receiver)

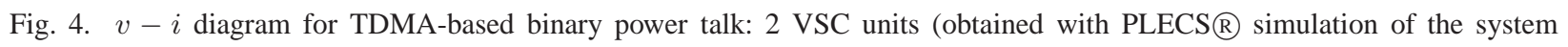
shown in Fig. $3 v^{0}=399 V, v^{1}=401 V, r_{d}^{0}=r_{d}^{1}=2 \Omega, v_{a}^{\mathrm{n}}=v_{b}^{\mathrm{n}}=400 V, r_{d, a}^{\mathrm{n}}=r_{d, b}^{\mathrm{n}}=2 \Omega$.)

Fig. 4[(a) illustrates detection spaces for VSCs in the example, assuming that VSC $a$ is active. If VSC $a$ inserts $\mathbf{x}^{0}$, it supplies less power than nominally, i.e., $P_{a}(0)<P_{a}^{\mathrm{n}}$. At the same time, VSC $b$ observes $\mathbf{s}_{b}(0)$, VSC $b$ detects that it supplies more than the nominal power $P_{b}(0)>P_{b}^{\mathrm{n}}$, and concludes that VSC $a$ is signaling " 0 ". Similarly, if VSC $a$ inserts $\mathbf{x}^{1}$, VSC $b$ observes $\mathbf{s}_{b}(1)$, detects that $P_{b}(1)<P_{b}^{\mathrm{n}}$, and concludes that VSC $a$ is signaling " 1 ". The TDMA scheme described above can be easily generalized to arbitrary number of units.

Full Duplex: In FD strategy, all units are simultaneously active. The same demodulation principle is applied: by observing its local output, a VSC can detect the information bit sent from the other VSC. The difference to the TDMA case is that the local output depends on the signaling combination of both VSCs:

$$
\mathbf{s}_{k}=\mathbf{s}_{k}\left(b_{a} b_{b}\right) \text { and } P_{k}=P_{k}\left(b_{a} b_{b}\right), k \in\{a, b\}
$$

as illustrated on Fig. 5 through the detection space for VSC $a$. Consider the case when VSC $a$ inserts $\mathbf{x}^{0}$, i.e., signals $b_{a}=0$. Then, depending on the symbol inserted by VSC $b$, VSC $a$ outputs different power $P_{a}$. In particular, for $b_{b}=0$, VSC $b$ outputs less power than nominally, while for $b_{b}=1$, VSC $b$ outputs more power than nominally; correspondingly, $P_{a}$ increases or decreases. The same reasoning applies when VSC $a$ inserts $\mathbf{x}^{1}$, as well as for the detection of $b_{a}$ at VSC $b$.

The two VSC unit example exposes two crucial issues. First, the described communication protocol requires prior knowledge of all possible points $\mathbf{s}_{k}$ in the detection space/local power 


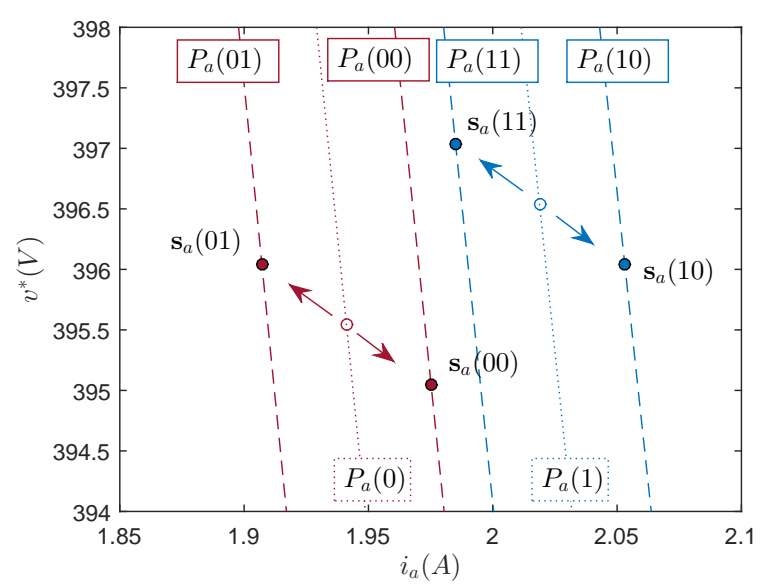

Fig. 5. $v-i$ diagram for FD-based binary power talk: 2 VSC units (obtained with PLECS $₫$ simulation of the system shown on Fig. 3, $v^{0}=399 V, v^{1}=401 V, r_{d}^{0}=r_{d}^{1}=2 \Omega, v_{a}^{\mathrm{n}}=v_{b}^{\mathrm{n}}=400 V, r_{d, a}^{\mathrm{n}}=r_{d, b}^{\mathrm{n}}=2 \Omega$.)

outputs $P_{k}$. As the detailed configuration of the MG is typically not known a priori, these values have to be learned in a predefined training phase, during which each VSC constructs the detection space. Clearly, TDMA and FD strategies differ in terms of the amount of information required to construct the detection space. FD requires longer training phases and this is addressed in detail in Section VI-B, here we only provide an illustration. In system with two units, in TDMA binary power talk each unit has to learn two separate points when the other VSC unit transmits, such that the total number of points is four. In FD binary power talk, each unit has to learn four points in its detection space, leading to a total of eight points for the system. Second, the output power of a VSC can also vary as a result of the load change, which happens arbitrarily and randomly. In particular, the current value of the load $r$ can be seen as a state of the system or the state of the communication channel. Whenever $r$ changes, the structure of the detection space also changes, leading to incorrect decisions at the receivers if the detection space prior to change is still used. A strategy to deal with random state variations is to periodically repeat the training phase or to provide mechanism that tracks the state changes and re-initiates the training phase whenever a change is detected. Section VI is dedicated to dealing with this challenge.

\section{B. DC MG system with three units: Characterization of Multiple Access}

Consider a DC MG system with three VSC units, denoted with $a, b$ and $c$, see Fig. 6, The units can communicate using TDMA or FD strategy. The TDMA solution, outlined in the previous 


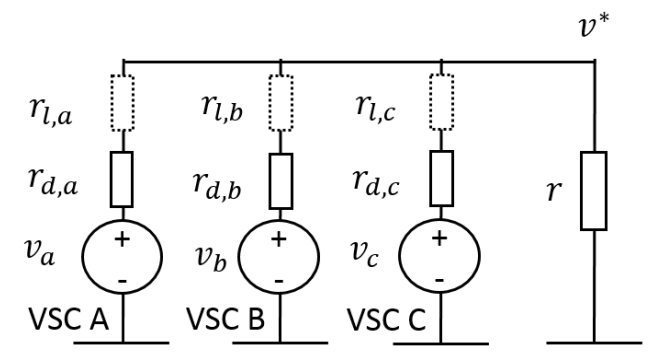

Fig. 6. DC MG system with three VSC units in steady-state.

example, can be straightforwardly generalized to arbitrary number of units in the system and the basic communication principles remain the same as illustrated in Fig. 4 . The FD power talk, however, faces an additional challenge. Assuming that the load is stable and applying the notation $\mathbf{s}_{k}=\mathbf{s}_{k}\left(b_{a} b_{b} b_{c}\right)$ and $P_{k}=P_{k}\left(b_{a} b_{b} b_{c}\right)$, VSC $a$ can observe the following symbols in the detection space $s_{a}$ /output powers $P_{a}$, as depicted in Fig. 77.

$$
\begin{aligned}
& \mathbf{s}_{a}(000) \leftrightarrow P_{a}(000) ; \quad \mathbf{s}_{a}(011) \leftrightarrow P_{a}(011) ; \quad \mathbf{s}_{a}(001) \cong \mathbf{s}_{a}(010) \leftrightarrow P_{a}(001) \cong P_{a}(010) \\
& \mathbf{s}_{a}(100) \leftrightarrow P_{a}(100) ; \quad \mathbf{s}_{a}(111) \leftrightarrow P_{a}(111) ; \quad \mathbf{s}_{a}(101) \cong \mathbf{s}_{a}(110) \leftrightarrow P_{a}(101) \cong P_{a}(110)
\end{aligned}
$$

Obviously, the outputs $\mathbf{s}_{a}(001)$ and $\mathbf{s}_{a}(010)$, as well as the outputs $\mathbf{s}_{a}(101)$ and $\mathbf{s}_{a}(110)$, are indistinguishable. In other words, VSC $a$ can not distinguish between the cases in which the sum of the bits of the other units is the same, as then the sum of their output powers is the same and, thus, the output power $P_{a}$ is the same. Also, it is easy to verify that the value of $\mathbf{s}_{a} / P_{a}$ depends on the bit signaled by VSC $a$ and the integer sum, i.e., the Hamming weight, of the bits signaled by the other units. Summarizing, the FD binary power talk system, as seen from each VSC locally and given the value of the local input, can be equivalently represented by a Multiple Access Adder Channel with Binary Inputs (BI-MAAC). This example illustrates another major difference between TDMA and FD power talk. Namely, in TDMA by demodulating the symbols in the detection space (see Fig. 4b) the information bit is directly obtained. FD power talk (see Fig. 7), in turn, calls for the corresponding BI-MAAC coding methods in order to obtain individual bit streams from the aggregate observations, i.e., the modulation and coding with FD are separated. Section $\mathrm{V}$ introduces the detection mechanism used for symbol demodulation for TDMA and FD, whereas multiple access coding for FD is treated in Section VI. 


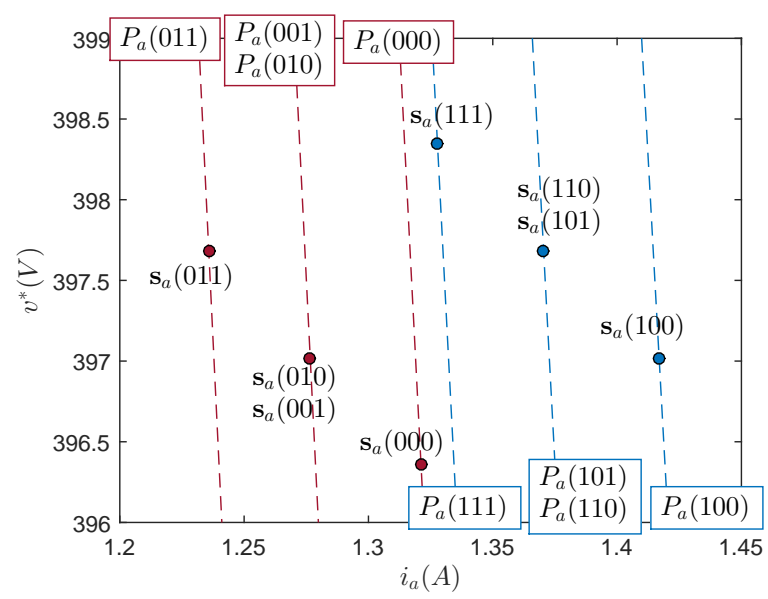

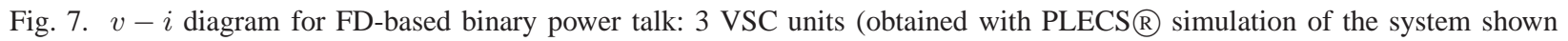
on Fig. 6 $v^{0}=399 V, v^{1}=401 V, r_{d}^{0}=r_{d}^{1}=2 \Omega, v_{a}^{\mathrm{n}}=v_{b}^{\mathrm{n}}=v_{c}^{\mathrm{n}}=400 V, r_{d, a}^{\mathrm{n}}=r_{d, b}^{\mathrm{n}}=r_{d, c}^{\mathrm{n}}=2 \Omega$.)

\section{General Binary Communication Model}

Consider a DC MG with $K$ units, connected in parallel to a common bus through feeder lines with negligible resistances, supplying resistive load $r$. Assume a binary communication scheme, in which units send $\mathbf{x}^{1}=\left(v^{1}, r_{d}^{1}\right)$ for bit value " 1 " and $\mathbf{x}^{0}=\left(v^{0}, r_{d}^{0}\right)$ for bit value “0”. Without loss of generality, assume that $P_{k}(1)>P_{k}(0), \forall r \in\left[R_{\min }, R_{\max }\right]$ where $P_{k}\left(b_{k}\right)$ is the output power of VSC $k$ when transmitting bit $b_{k}$ and all other units operate in nominal mode. The output of unit $k$ is $\mathbf{s}_{k}=\left(v^{*}, i_{k}\right)$, the output consists of the bus voltage $v^{*}$ and output current $i_{k}$, which determine the output power $P_{k}$. As noted above, $\mathbf{s}_{k}$ and, thus, $P_{k}$ depend on the inputs of all units, not just unit $k$, as well as on the value of the load $r$. All units measure locally their outputs, i.e., unit $k$ observes its $\mathbf{s}_{k}$, based on which detection of the symbols of the active, signaling units is performed. The observation of $\mathbf{s}_{k}$, denoted by $\mathbf{y}_{k}=\left(\tilde{v}^{*}, \tilde{i}_{k}\right)$, differs from $\mathbf{s}_{k}=\left(v^{*}, i_{k}\right)$ due to voltage and current uncertainties caused by [19]-[21]: i) parasitic effects in the integrated circuits, ii) inaccuracies of the pulse width modulation process, iii) electromagnetic interference due to intentional/unintentional emissions by circuits in the system and from the surroundings, iv) ambient temperature, which is known to alter the performance of the power electronic components and v) sensor and measurement noise. As suggested in [22], [23] and references therein, the aggregate uncertainty of the measurements in the feedback loops of the primary control, after averaging with LPF, can be accurately modeled with a Gaussian noise:

$$
\mathbf{y}_{k}=\left(\tilde{v}^{*}, \tilde{i}_{k}\right)=\mathbf{s}_{k}+\mathbf{z}_{k}
$$




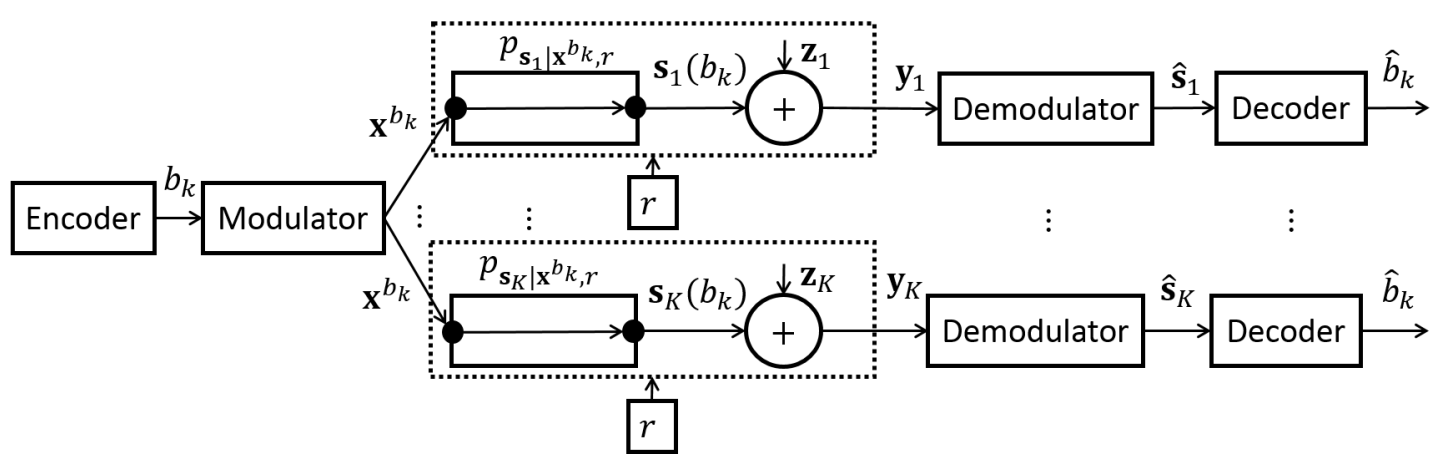

Fig. 8. Communication model for TDMA-based binary power talk: VSC $k$ as a transmitter.

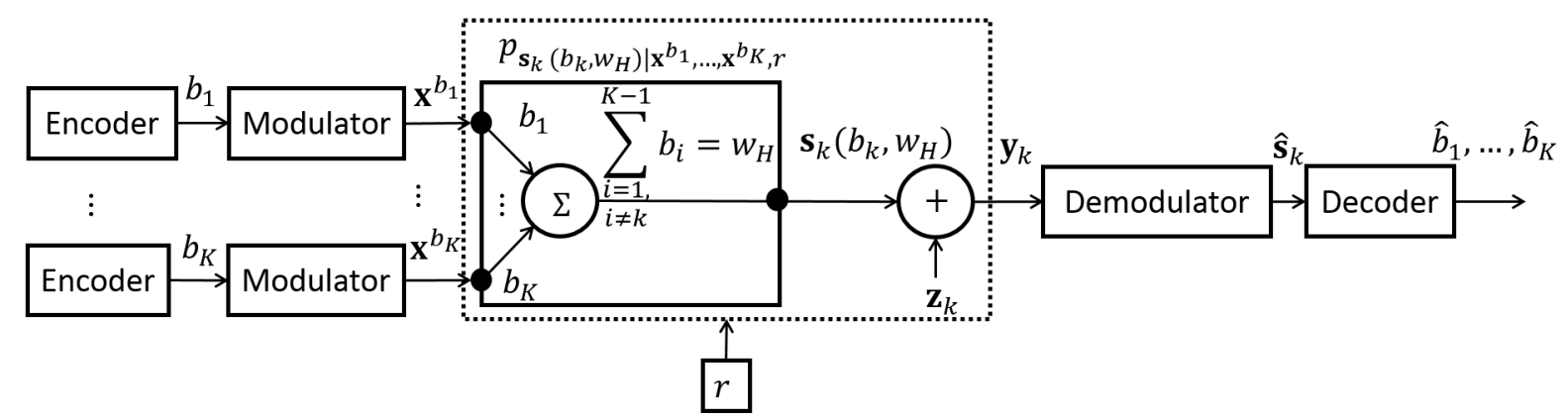

Fig. 9. General Communication model for FD-based binary power talk: VSC $k$ as a receiver.

where $\mathbf{z}_{k} \sim \mathcal{N}\left(\mathbf{0}, \operatorname{diag}\left\{\sigma_{v^{*}}^{2}, \sigma_{i_{k}}^{2}\right\}\right)$. Therefore, the observation $\mathbf{y}_{k}$, given the momentary value of the load $r$, will be distributed according to the Gaussian conditional pdf $p\left(\mathbf{y}_{k} \mid \mathbf{s}_{k}, r\right)$.

For TDMA power talk, a unit transmits only in time slots exclusively dedicated to it, while the rest of the units operate in the nominal mode. Therefore, in every time slot, the MG can be equivalently represented as a broadcast channel from the signaling unit to all other units, as depicted on Fig. 8. When VSC $k$ transmits bit $b_{k}$, its input symbol $\mathbf{x}^{b_{k}}$ maps to output $\mathbf{s}_{j}\left(b_{k}\right)$ at VSC $j, j=1, \ldots, K$. In turn, VSC $j$ observes $\mathbf{y}_{j}=\mathbf{s}_{j}\left(b_{k}\right)+\mathbf{z}$, and makes decision $\hat{b}_{k}$.

For FD power talk, all units transmit in every time slot, and the local observation of the output $\mathbf{y}_{k}, k=1, \ldots, K$, depends on the information bits of all units. As illustrated in Section ఋII-B, the equivalent channel model, seen from each unit locally, can be represented by BI-MAAC with $K-1$ users, see Fig. 9. The local output $\mathbf{s}_{k}$ depends on the combination of $b_{k}$ and $w_{H}\left(\mathbf{b}_{\sim k}\right)$, where $w_{H}$ denotes Hamming weight and $\mathbf{b}_{\sim k}$ is the sequence of bits of all units except VSC $k$ :

$$
\mathbf{s}_{k}=\mathbf{s}_{k}\left(b_{k}, w_{H}\left(\mathbf{b}_{\sim k}\right)\right) \text { and } P_{k}=P_{k}\left(b_{k}, w_{H}\left(\mathbf{b}_{\sim k}\right)\right), k=1, \ldots, K, .
$$


The number of outputs that VSC $k$ may observe locally is $2 K$, i.e., $K$ points for each value of the local bit $b_{k}$. Also, for given $r$, it can be shown that the output powers at VSC $k, k=1, \ldots, K$ satisfy $P_{k}\left(b_{k}, W_{1}\right)>P_{k}\left(b_{k}, W_{2}\right)$ if $W_{1}<W_{2}$, and where $W_{1}, W_{2}=0, \ldots, K-1$ are Hamming weights, see (14).

\section{Communication under Constraints: Signaling And Detection Spaces}

Here we introduce the concept of signaling space to capture the effect that the operating constraints of the MG have on the power talk schemes. We then investigate the structure of the detection space and its dynamics due to the load changes, as this is vital for implementing reliable power talk solutions.

\section{A. The signaling space}

Every MG is subject to operational constraints that may not be violated. Denote by $\mathcal{C}$ a set that consists of all operational constraints. The signaling space $\mathcal{X}$ is the set of all possible symbols $\mathbf{x}_{k}, k=1, \ldots, K$, that jointly satisfy the constraints in $\mathcal{C}$ for any value $r \in\left[R_{\text {min }}, R_{\text {max }}\right]$ :

$$
\mathcal{X}=\left\{\mathbf{x}_{k}=\left(v_{k}, r_{d, k}\right), k=1, \ldots, K: \mathcal{C}, r \in\left[R_{\text {min }}, R_{\text {max }}\right]\right\}
$$

In general, $\mathcal{C}$ comprises constraints associated with system stability and power delivery quality, e.g., limits on the voltage, current, power dissipation, droop slope etc. In this paper, we focus on the most important constraints, namely the bus voltage and output current constraints:

$$
\mathcal{C}=\left\{V_{\min } \leq v^{*} \leq V_{\max } ; I_{k, \min } \leq i_{k} \leq I_{k, \max }, k=1, \ldots, K\right\},
$$

where $V_{\min }$ and $V_{\max }$ are the minimum and maximum allowable bus voltages and where $I_{k, \min }$ and $I_{k, \max }$ are the minimum and maximum output currents of VSC $k$; usually, $I_{k, \min }=0$ and $I_{k, \max }$ is the current rating of the unit.

In this paper, we deal with binary power talk when all units employ the same symbols, i.e., $\mathbf{x}_{k}^{1}=\mathbf{x}^{1}=\left(v^{1}, r_{d}^{1}\right)$ and $\mathbf{x}_{k}^{0}=\mathbf{x}^{0}=\left(v^{0}, r_{d}^{0}\right)$. Then, in the TDMA case, under constraints (16) and the steady-state model (2) and (3), the symbols $\mathrm{x}^{1}$ and $\mathrm{x}^{0}$ should satisfy:

$$
\begin{gathered}
V_{\min }-\frac{\sum_{i \neq k} \frac{v_{i}^{\mathrm{n}}}{r_{d, i}^{n}}}{\left(\frac{1}{R_{\min }}+\sum_{i \neq k} \frac{1}{r_{d, j}^{n}}\right)} \\
r_{d}^{b} \frac{V_{\max }-\frac{\sum_{i \neq k} \frac{v_{i}^{\mathrm{n}}}{r_{d, i}^{n}}}{\left(\frac{1}{R_{\max }}+\sum_{i \neq k} \frac{1}{r_{d, i}^{n}}\right)}}{\left(\frac{1}{R_{\min }}+\sum_{i \neq k} \frac{1}{r_{d, i}^{\mathrm{n}}}\right)^{-1}}+V_{\min } \leq v^{b} \leq r_{d}^{b} \frac{1}{\left(\frac{1}{R_{\max }}+\sum_{i \neq k} \frac{1}{r_{d, i}^{\mathrm{n}}}\right)^{-1}}, \\
\frac{\sum_{i \neq k} \frac{v_{i}^{\mathrm{n}}}{r_{d, i}^{\mathrm{n}}}}{\left(\frac{1}{R_{\max }}+\sum_{i \neq k} \frac{1}{r_{d, i}^{\mathrm{n}}}\right)} \leq v^{b} \leq r_{d}^{b} I_{k, \max }+\frac{I_{k, \max }}{\left(\frac{1}{R_{\min }}+\sum_{i \neq k} \frac{1}{r_{d, i}^{\mathrm{n}}}\right)}+\frac{\sum_{i \neq k}^{\mathrm{n}}}{\left(\frac{1}{r_{d, i}^{\mathrm{n}}}+\sum_{i \neq k} \frac{1}{r_{d, i}^{n}}\right)},
\end{gathered}
$$




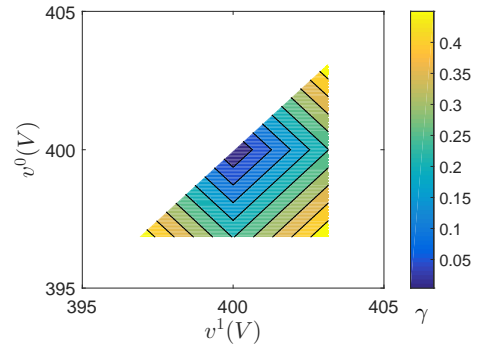

(a) TDMA: $K=2$

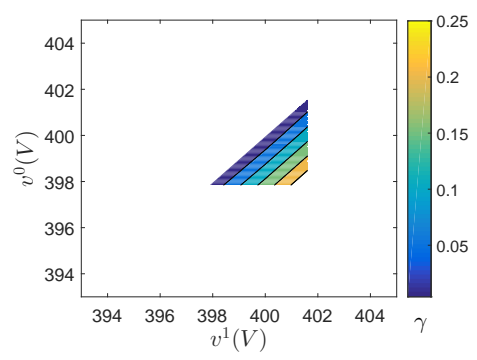

(d) FD: $K=2$

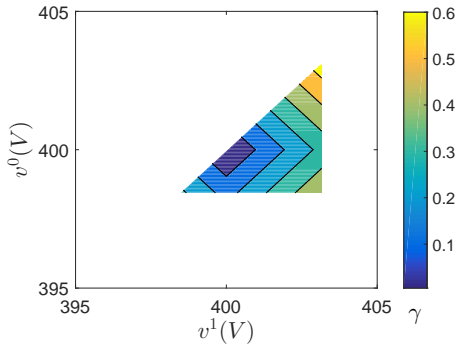

(b) TDMA: $K=3$

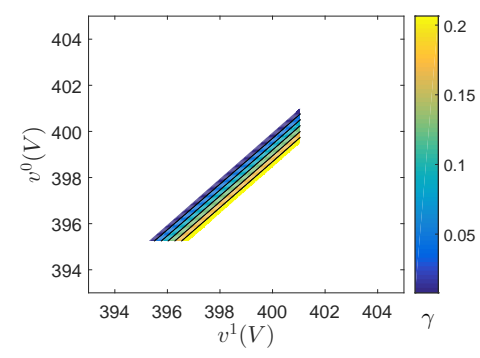

(e) FD: $K=3$

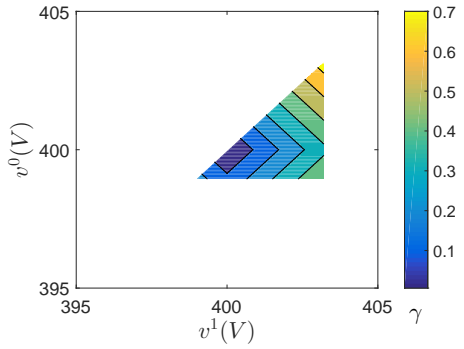

(c) TDMA: $K=4$

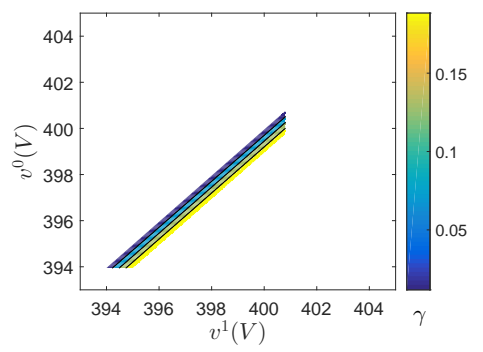

(f) FD: $K=4$

Fig. 10. The signaling space and average power deviation. Fixed $r_{d, a}$ constellation, $r_{d}^{1}=r_{d}^{0}=2 \Omega$. Equiprobable bit values.

for $k=1, \ldots, K$ and $b \in\{0,1\}$. In FD case, all units simultaneously change their droop parameters and it can be shown that $\mathbf{x}^{1}$ and $\mathbf{x}^{0}$ should satisfy $(k=1, \ldots, K$ and $b \in\{0,1\})$ :

$$
\begin{aligned}
r_{d}^{b} \frac{V_{\min }}{K R_{\min }}+V_{\min } \leq v^{b} \leq r_{d}^{b} \frac{V_{\max }}{K R_{\max }}+V_{\max }, \\
\quad \frac{(K-1) \frac{v^{0}}{r_{d}^{0}}}{\left(\frac{1}{R_{\min }}+\frac{K-1}{r_{d}^{0}}\right)} \leq v^{1} \leq I_{k, \max } r_{d}^{1}+\frac{I_{k, \max }}{\left(\frac{1}{R_{\min }}+\frac{K-1}{r_{d}^{0}}\right)}+\frac{(K-1) \frac{v^{0}}{r_{d}^{0}}}{\left(\frac{1}{R_{\min }}+\frac{K-1}{r_{d}^{0}}\right)}, \\
\quad \frac{(K-1) \frac{v^{1}}{r_{d}^{1}}}{\left(\frac{1}{R_{\max }}+\frac{K-1}{r_{d}^{1}}\right)} \leq v^{0} \leq I_{k, \max } r_{d}^{0}+\frac{I_{k, \max }}{\left(\frac{1}{R_{\max }}+\frac{K-1}{r_{d}^{1}}\right)}+\frac{(K-1) \frac{v^{1}}{r_{d}^{1}}}{\left(\frac{1}{R_{\max }}+\frac{K-1}{r_{d}^{1}}\right)} .
\end{aligned}
$$

Each $\mathrm{x}_{k} \in \mathcal{X}$ results in different output power $P_{k}$. To account for this effect, we introduce the relative power deviation of unit $k$ w.r.t. its output power $P_{k}^{\mathrm{n}}$ when all units operate nominally:

$$
\delta_{k}\left(\mathbf{x}_{1}, \ldots, \mathbf{x}_{K}\right)=\frac{\sqrt{\mathbb{E}_{R}\left\{\left(P_{k}-P_{k}^{\mathrm{n}}\right)^{2}\right\}}}{\mathbb{E}_{R}\left\{P_{k}^{\mathrm{n}}\right\}}, k=1, \ldots, K
$$

where $\mathrm{x}_{1}, \ldots, \mathrm{x}_{K}$ are the input symbols of all units in the system (recall that output power of any unit depends on all inputs in the system), and where the averaging is performed over the 
load $r$, modeled as a random variable with distribution $R \sim p_{R}(r)$. The average relative power deviation of VSC $k$, and the average relative power deviation per VSC are simply:

$$
\begin{aligned}
\delta_{k} & =\mathbb{E}_{\mathbf{X}_{1}, \ldots, \mathbf{X}_{K}}\left\{\delta_{k}\left(\mathbf{x}_{1}, \ldots, \mathbf{x}_{K}\right)\right\}, \\
\delta & =\frac{1}{K} \sum_{k=1}^{K} \delta_{k},
\end{aligned}
$$

where the averaging in (23) is performed over the combinations of all input symbols. Again, we point out that in the TDMA case, only a single input in $\mathbf{x}_{1}, \ldots, \mathbf{x}_{K}$ represents an actual power talk symbol of the active unit, while the rest of them are nominal inputs. In FD case, all inputs represent power talk symbols. Finally, we introduce average power deviation limit $\gamma$, requiring that:

$$
\delta \leq \gamma
$$

i.e., the average power deviation per unit w.r.t. the nominal mode of operation is bound by $\gamma$. In TDMA case, (25) translates to an individual constraint, limiting the amount of output power deviation of each unit, whereas in FD case, it limits the average deviation of the power supplied to the load by all units jointly.

Fig. 10 shows the signaling space for binary power talk, for system parameters listed in Table II uniform distribution of the load $R \sim \mathcal{U}\left[R_{\min }, R_{\text {max }}\right]$, when both symbols are equiprobable and have fixed droop slope $r_{d}^{1}=r_{d}^{0}$ (also referred to as the fixed $r_{d}$ constellation) and $v^{1}>v^{0}$. Evidently, TDMA power talk offers larger signaling spaces for given $\gamma$. For both TDMA and $\mathrm{FD}$, the signaling spaces decrease as the number of units increases.

\section{B. The detection space}

As already introduced, the detection space $\mathcal{S}_{k}$ for VSC $k$ is defined as the set of points $\mathbf{s}_{k}=\left(v^{*}, i_{k}\right)$, where $v^{*}$ is the output voltage, equal to the bus voltage when the line resistance is negligible, and $i_{k}$ is the output current of VSC $k$. Physically, each point $\mathbf{s}_{k}$ represents the output power $P_{k}$. By observing $\mathbf{s}_{k}$, VSC $k$ gathers information about the symbols/powers of other units.

We start by outlining the general structure of the detection space, illustrated on Fig. 11, All possible outputs of VSC $k$ lie on the line $v^{*}=-r_{d, k} i_{k}+v_{k}$ where $\left(v_{k}, r_{d, k}\right)$ is the symbol VSC $k$ is inserting. For TDMA power talk, if VSC $k$ is receiving, then $\mathbf{x}_{k}=\mathbf{x}_{k}^{\mathrm{n}}=\left(v_{k}^{\mathrm{n}}, r_{d, k}^{\mathrm{n}}\right)$ and the output symbols lie on a single line, as shown in Figs. 11(a)-(c). For FD power talk, a VSC sends either $\mathrm{x}^{0}$ or $\mathrm{x}^{1}$, and there are two lines on which the outputs may lie, as shown in Figs. 11(d)-(f). 


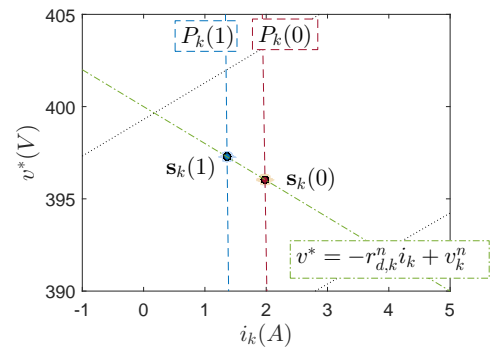

(a) TDMA: $K=2, r=100 \Omega$

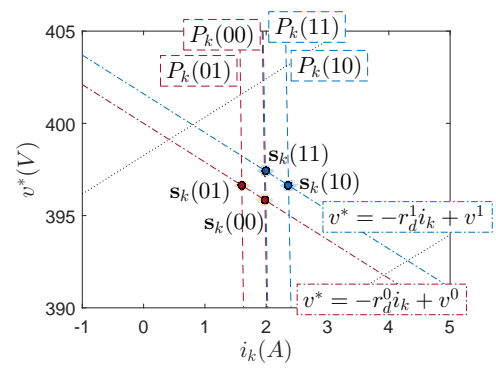

(d) FD: $K=2, r=100 \Omega$

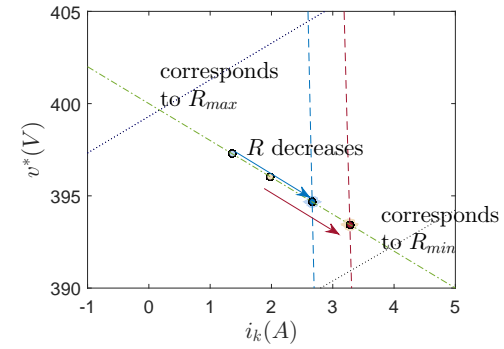

(b) TDMA: $K=2, r=60 \Omega$

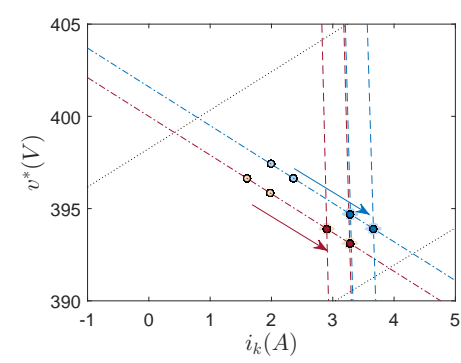

(e) FD: $K=2, r=60 \Omega$

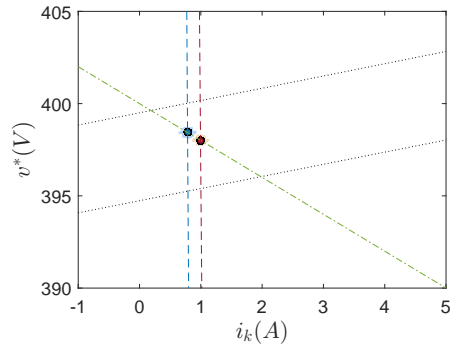

(c) TDMA: $K=4, r=100 \Omega$

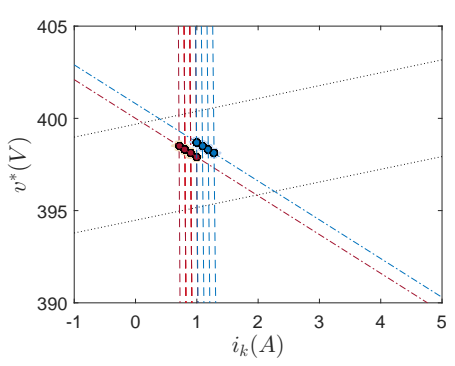

(f) FD: $K=4, r=100 \Omega$

Fig. 11. The detection space of VSC $k$, fixed $r_{d}$ constellation, $\gamma=0.2$. The dashed lines represent the output power $P_{k}=v^{*} i_{k}$ and the dashed-dotted line $v^{*}=-r_{d, k} i_{k}+v_{k}$ represents the symbol $\mathbf{x}_{k}$ that VSC $k$ is inserting. The loci of output symbols $\mathbf{s}_{k}$ are on the intersection between the lines corresponding to output powers and the local inputs. As $r$ varies, $\mathbf{s}_{k}$ slide along the dashed-dotted lines between the bounds (dotted lines) defined by operational constraints on the load $R_{\min }$ and $R_{\max }$.

Further, in both cases the actual loci of output symbols of VSC $k$, i.e., $\mathbf{s}_{k}=\left(v^{*}, i_{k}\right)$, are in the intersection of $v^{*}=-r_{d, k} i_{k}+v_{k}$ and $v^{*}=\frac{P_{k}}{i_{k}}$, where $P_{k}$ is the output power. As the load in the system varies, the points $\mathbf{s}_{k}$ in $\mathcal{S}_{k}$ shift along $v^{*}=-r_{d, k} i_{k}+v_{k}$, see Fig. 11(b) and Fig. 11(e). Comparing Fig. 11(c) and Fig. 11(f) with Fig. 11(a) and Fig. 11(d), respectively, it is apparent that under constant average power deviation constraint, increasing the number of units shrinks the detection space and reduces the distance among the symbols, making the detection more susceptible to noise. This effect is more evident for FD power talk.

Next, we describe the detection mechanism. We assume that all VSCs, when transmitting, behave as i.i.d. Bernoulli sources, with probability of transmitting "1" denoted by $p_{b}$. Each VSC obtains a noisy observation $\mathbf{y}_{k}=\left(\tilde{v}^{*}, \tilde{i}_{k}\right), k=1, \ldots, K$, see (13). In TDMA binary power talk, VSC $k$ should decide between $\mathbf{s}_{k}(1)=\left(v^{*}(1), i_{k}(1)\right)$ and $\mathbf{s}_{k}(0)=\left(v^{*}(0), i_{k}(0)\right)$ based on $\mathbf{y}_{k}$, and for this purpose, employs Maximum A Posteriori Detection (MAPD) under Gaussian noise:

$$
\ln \frac{p\left(\mathbf{y}_{k} \mid \mathbf{s}_{k}(1), r\right)}{p\left(\mathbf{y}_{k} \mid \mathbf{s}_{k}(0), r\right)} \underset{\mathbf{s}_{k}(0)}{\gtrless} \sin \frac{1-p_{b}}{p_{b}} .
$$


The decision regions, i.e., the sets of points satisfying (26), are:

$$
\begin{aligned}
& \Lambda_{1}(r): \tilde{v}^{*}>\tilde{i}_{k} a_{k}^{1,0}+b_{k}^{1,0}, \text { for } \mathbf{s}_{k}(1), \\
& \Lambda_{0}(r): \tilde{v}^{*}<\tilde{i}_{k} a_{k}^{1,0}+b_{k}^{1,0}, \text { for } \mathbf{s}_{k}(0),
\end{aligned}
$$

where:

$$
\begin{aligned}
a_{k}^{1,0} & =\frac{\sigma_{v^{*}}^{2}}{\sigma_{i_{k}}^{2}} \frac{i_{k}(1)-i_{k}(0)}{v^{*}(0)-v^{*}(1)} \\
b_{k}^{1,0} & =\frac{1}{2}\left(v^{*}(0)+v^{*}(1)\right)+\frac{1}{2} \frac{\sigma_{v^{*}}^{2}}{\sigma_{i_{k}}^{2}} \frac{\left(i_{k}(0)\right)^{2}-\left(i_{k}(1)\right)^{2}}{v^{*}(0)-v^{*}(1)}+\frac{\sigma_{v^{*}}^{2}}{v^{*}(1)-v^{*}(0)} \ln \frac{1-p_{b}}{p_{b}}
\end{aligned}
$$

The error probabilities, given that the true outputs are $\mathbf{s}_{k}(1)$ and $\mathbf{s}_{k}(0)$ are, respectively:

$$
\begin{array}{r}
\operatorname{Pr}\left(e_{k} \mid \mathbf{s}_{k}(1), r\right)=\int_{\mathbf{y}_{k} \in \Lambda_{0}(r)} p\left(\mathbf{y}_{k} \mid \mathbf{s}_{k}(1), r\right) d \tilde{v}^{*} d \tilde{i}_{k}=1-Q\left(\frac{b_{k}^{1,0}-v^{*}(1)+i_{k}^{1} a_{k}^{1,0}}{\sqrt{\sigma_{v^{*}}^{2}+\left(\sigma_{i_{k}} a_{k}^{1,0}\right)^{2}}}\right) \\
\operatorname{Pr}\left(e_{k} \mid \mathbf{s}_{k}(0), r\right)=\int_{\mathbf{y}_{k} \in \Lambda_{1}(r)} p\left(\mathbf{y}_{k} \mid \mathbf{s}_{k}(0), r\right) d \tilde{v}^{*} d \tilde{i}_{k}=Q\left(\frac{b_{k}^{1,0}-v^{*}(0)+i_{k}^{0} a_{k}^{1,0}}{\sqrt{\sigma_{v^{*}}^{2}+\left(\sigma_{i_{k}} a_{k}^{1,0}\right)^{2}}}\right)
\end{array}
$$

Finally, the average error probability is:

$$
\operatorname{Pr}\left(e_{k}\right)=\mathbb{E}_{R}\left\{\operatorname{Pr}\left(e_{k} \mid \mathbf{s}_{k}(1), r\right) p_{b}+\operatorname{Pr}\left(e_{k} \mid \mathbf{s}_{k}(0), r\right)\left(1-p_{b}\right)\right\}
$$

In FD binary power talk, VSC $k$ receives $\mathbf{s}_{k}\left(b_{k}, w_{H}\right)=\left(v^{*}\left(b_{k}, w_{H}\right), i_{k}\left(b_{k}, w_{H}\right)\right)$ where $w_{H}\left(\mathbf{b}_{\sim k}\right)$ is the Hamming weight of the sequence of bits $\mathbf{b}_{\sim k}$ of the other units, see (14). Given the local input $\mathbf{x}^{b_{k}}, \mathbf{s}_{k}\left(b_{k}, w_{H}\right)$ is a priori distributed according to the Binomial distribution:

$$
\operatorname{Pr}\left(\mathbf{s}_{k}\left(b_{k}, w_{H}\right)\right)=\left(\begin{array}{c}
K-1 \\
w_{H}
\end{array}\right) p_{b}^{w_{H}}\left(1-p_{b}\right)^{K-1-w_{H}}
$$

The MAPD decides in favor of $\mathbf{s}_{k}\left(b_{k}, W_{i}\right)$ when $\mathbf{y}_{k}=\left(\tilde{v}^{*}, \tilde{i}_{k}\right)$ is observed if:

$$
\ln \frac{p\left(\mathbf{y}_{k} \mid \mathbf{s}_{k}\left(b_{k}, W_{i}\right), r\right)}{p\left(\mathbf{y}_{k} \mid \mathbf{s}_{k}\left(b_{k}, W_{j}\right), r\right)} \geq \ln \frac{\operatorname{Pr}\left(\mathbf{s}_{k}\left(b_{k}, W_{j}\right)\right)}{\operatorname{Pr}\left(\mathbf{s}_{k}\left(b_{k}, W_{i}\right)\right)}, W_{j}=0, \ldots, K-1, W_{i} \neq W_{j}
$$

The decision region for symbol $\mathbf{s}_{k}\left(b_{k}, W_{i}\right)$, denoted by $\Lambda_{W_{i}}(r)$, is defined as:

$$
\Lambda_{W_{i}}(r):\left\{\begin{array}{l}
\tilde{v}^{*}<\tilde{i}_{k} a_{k, b_{k}}^{W_{i}+1, W_{i}}+b_{k, b_{k}}^{W_{i}+1, W_{i}} \\
\tilde{v}^{*}>\tilde{i}_{k} a_{k, b_{k}}^{W_{i}-1, W_{i}}+b_{k, b_{k}}^{W_{i}-1, W_{i}}
\end{array}\right.
$$

for $0<W_{i}<K-1$ and:

$$
\begin{aligned}
\Lambda_{0}(r): & \tilde{v}^{*}<\tilde{i}_{k} a_{k, b_{k}}^{1,0}+b_{k, b_{k}}^{1,0}, \\
\Lambda_{K-1}(r): & \tilde{v}^{*}>\tilde{i}_{k} a_{k, b_{k}}^{K-1, K-2}+b_{k, b_{k}}^{K-1, K-2} .
\end{aligned}
$$


for $\mathbf{s}_{k}\left(b_{k}, 0\right)$ and $\mathbf{s}_{k}\left(b_{k}, K-1\right)$, respectively, where:

$$
\begin{aligned}
a_{k, b_{k}}^{l, h}= & \frac{\sigma_{v^{*}}^{2}}{\sigma_{i_{k}}^{2}} \frac{i_{k}\left(b_{k}, l\right)-i_{k}\left(b_{k}, h\right)}{v^{*}\left(b_{k}, h\right)-v^{*}\left(b_{k}, l\right)} \\
b_{k, b_{k}}^{l, h}= & \frac{1}{2}\left(v^{*}\left(b_{k}, h\right)+v^{*}\left(b_{k}, l\right)\right)+\frac{1}{2} \frac{\sigma_{v^{*}}^{2}}{\sigma_{i_{k}}^{2}} \frac{\left(i_{k}\left(b_{k}, h\right)\right)^{2}-\left(i_{k}\left(b_{k}, l\right)\right)^{2}}{v^{*}\left(b_{k}, h\right)-v^{*}\left(b_{k}, l\right)}+ \\
& +\frac{\sigma_{v^{*}}^{2}}{v^{*}\left(b_{k}, h\right)-v^{*}\left(b_{k}, l\right)} \ln \frac{\operatorname{Pr}\left(\mathbf{s}_{k}\left(b_{k}, l\right)\right)}{\operatorname{Pr}\left(\mathbf{s}_{k}\left(b_{k}, h\right)\right)}
\end{aligned}
$$

and $l, h=0, \ldots, K-1, l \neq h$. The probability of error when the true output is $\mathbf{s}_{k}\left(b_{k}, W_{i}\right)$ is:

$$
\operatorname{Pr}\left(e_{k} \mid \mathbf{s}_{k}\left(b_{k}, W_{i}\right), r\right)=\int_{\mathbf{y}_{k} \notin \Lambda_{W_{i}}(r)} p\left(\mathbf{y}_{k} \mid \mathbf{s}_{k}\left(b_{k}, W_{i}\right), r\right) d \tilde{v}^{*} d \tilde{i}_{k}
$$

and the average error probability can be calculated as:

$$
\operatorname{Pr}\left(e_{k}\right)=\mathbb{E}_{R}\left\{\sum_{\mathbf{x}^{b} \in\left\{\mathbf{x}^{1}, \mathbf{x}^{0}\right\}} \sum_{W_{i}=0}^{K-1} \operatorname{Pr}\left(e_{k} \mid \mathbf{s}_{k}\left(b_{k}, W_{i}\right), r\right)\left(\begin{array}{c}
K-1 \\
W_{i}
\end{array}\right) p_{b}^{W_{i}}\left(1-p_{b}\right)^{K-1-W_{i}} \operatorname{Pr}\left(\mathbf{x}^{b_{k}}\right)\right\}
$$

As already noted, the observations $\mathbf{y}_{k}, k=1, \ldots, K$, are obtained as averages over a slot. The variance of the observation noise $\sigma^{2}=\operatorname{diag}\left\{\sigma_{v^{*}}^{2}, \sigma_{i_{k}}^{2}\right\}$, see Section IV depends on the slot duration $T_{s}$ and the sampling frequency $f_{o}$. As $T_{s}$ is of the order of milliseconds and $f_{s}$ of the order of $\mathrm{kHz}, \sigma_{v^{*}}^{2}$ and current $\sigma_{i_{k}}^{2}$ have rather modest values. Fig. 12 illustrates the probability of correct detection $P_{D}=1-\frac{1}{K} \sum_{k=1}^{K} P\left(e_{k}\right)$ as the number of units in the system $K$ increases. The values $\sigma_{v^{*}}=\sigma_{i_{k}}=0.001$ corresponding to the worst case scenario of the observation noise variance for the measurement equipment used in modern low voltage MGs [19]-[23], when $T_{s}=1 \mathrm{~ms}$ and $f_{o}=10 \mathrm{kHz}$. The symbols are chosen from the fixed $r_{d}$ constellation such that the average relative power deviation $\gamma=0.05$ is satisfied, i.e., the constellation is not specifically optimized to minimize the error probability. Obviously, the proposed detector performs exceptionally well in case of TDMA power talk. In FD version of the scheme, the detection is also practically errorless for $K \leq 8$. We also note that for larger $\gamma$ the effects of noise become practically negligible both for TDMA and FD binary power talk. Taking into account the above results, it is evident that the need for the additional mechanisms to combat observation noise is rather modest, if the proposed detection method is employed.

We conclude by noting that the decision regions are established for a given value of the load $r$, and they need to be reset when $r$ changes. This could done using training sequences, which are sent by VSCs in a coordinated manner, and which enable construction of $\mathcal{S}_{k}, k=1, \ldots, K$. Details on this aspect of power talk are given in the next section. 


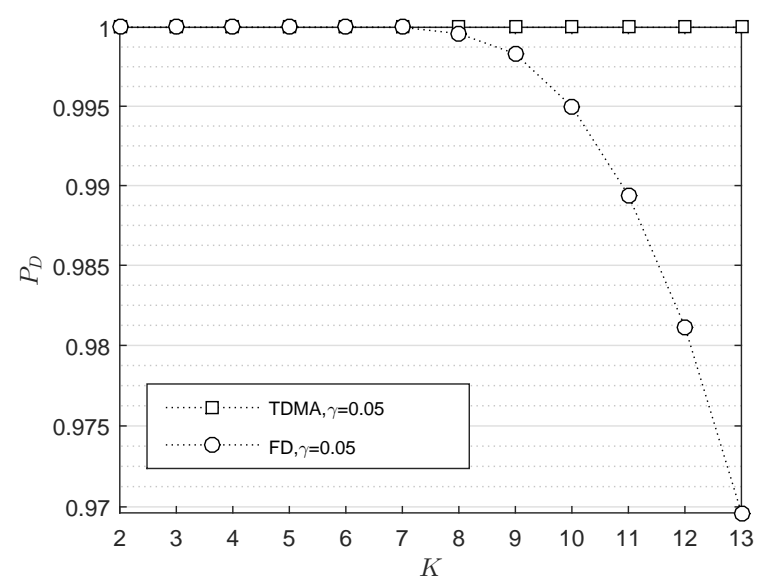

Fig. 12. Probability of correct detection. Fixed $r_{d}$ constellation, $r_{d}^{1}=r_{d}^{0}=2 \Omega, v^{0}=400 V, v^{1}$ chosen to satisfy $\delta=\gamma$, equiprobable bit values.

\section{Vi. Designing Power Talk Communication Protocols}

In this section, we present techniques that complement the basic power talk operation, as described in the previous Sections, required to design a fully operational communication protocol. To evaluate the efficiency of the proposed solutions, we use the net transmission rate per unit $\eta$, i.e., average number of information bits transmitted per unit per time slot. In further text, we assume a MG with $K$ units and fair scheduling.

\section{A. Coding for multiple access}

Here we compare the rates of TDMA and FD versions of power talk, denoted by $\eta_{\text {TDMA }}^{\mathrm{S}}$ and $\eta_{\mathrm{FD}}^{\mathrm{S}}$, respectively when $r$ is stable, i.e., it does not change, and the detection spaces are accurate. In TDMA power talk, the transmissions are orthogonal in time and the net transmission rate can be simply written as:

$$
\eta_{\mathrm{TDMA}}^{\mathrm{S}}=1 / K .
$$

In FD power talk, we deal with BI-MAAC, where a unit observes sums of the bits transmitted by $K-1$ units. Chang and Weldon in [24] proposed a reference coding solution for this type of multiple access, which enables unique decodability of user codewords and asymptotically achieves the maximum sum rate of the BI-MAAC. Table $[$ lists the achievable transmission 
TABLE II

ACHIEvable RATes of CHANG-Weldon COdes [24] FOR BI-MAAC

\begin{tabular}{|c||c|c|c|c|c|c|c|c|c|c|c|c|c|c|c|c|c|c|}
\hline$K$ & 1 & 2 & 3 & 4 & 5 & 6 & 7 & 8 & 9 & 10 & 11 & 12 & 13 & 14 & 15 & 16 & 17 & 18 \\
\hline$\eta_{\mathrm{FD}}^{S}$ & 1 & $1 / 2$ & $1 / 2$ & $1 / 3$ & $1 / 3$ & $1 / 4$ & $1 / 4$ & $1 / 4$ & $1 / 5$ & $1 / 5$ & $1 / 6$ & $1 / 6$ & $1 / 6$ & $1 / 7$ & $1 / 7$ & $1 / 8$ & $1 / 8$ & $1 / 8$ \\
\hline
\end{tabular}

rates per unit per slot $\eta_{\mathrm{FD}}^{S}$ for the scheme from [24], again assuming stable operation. Evidently, from Table $\llbracket$ it follows:

$$
\eta_{\mathrm{FD}}^{\mathrm{S}}>\eta_{\mathrm{TDMA}}^{\mathrm{S}}=1 / K
$$

Therefore, FD power talk is more efficient in terms of resource utilization, when the effects related to re-establishment of the detection space when the changes of the load $r$ are neglected.

\section{B. Training phase}

All VSCs have to maintain a layout of the detection space that matches the current value of the load $r$. A simple and effective strategy for the construction of the detection space is to conduct a training phase during which the units input predefined training sequences. We assume that each unit builds its detection space separately, in order to take into account imperfections of the MG system, such as small resistances of the feeder lines and the common bus.

Denote the length of the training phase in slots by $L$. In TDMA binary power talk, a unit has to learn 2 points in its detection space for each of the remaining units, see Section III. Assuming that a point is learned during $M$ dedicated slots 2 the training phase length is $L_{\mathrm{TDMA}}=2 M K$ slots. The training phase can be performed by sequential transmission of $\mathrm{x}^{0}$ and $\mathrm{x}^{1}$ by one of the units, while the remaining units operate nominally and construct their detection spaces.

In FD-based power talk, the number of outputs each VSC can observe in the detection space is $2 K$, see Section 【II. Then, the total number of points to be learned is $2 K^{2}$, and, assuming that a point is learned during $M$ slots, the training phase length is $L_{\mathrm{FD}}=2 M K^{2}$. The training can be conducted such that a units construct their detection spaces sequentially, through coordinated

\footnotetext{
${ }^{2}$ In general, using $M>1$ improves the reliability of the detection space construction, at a cost of an increased training phase.
} 


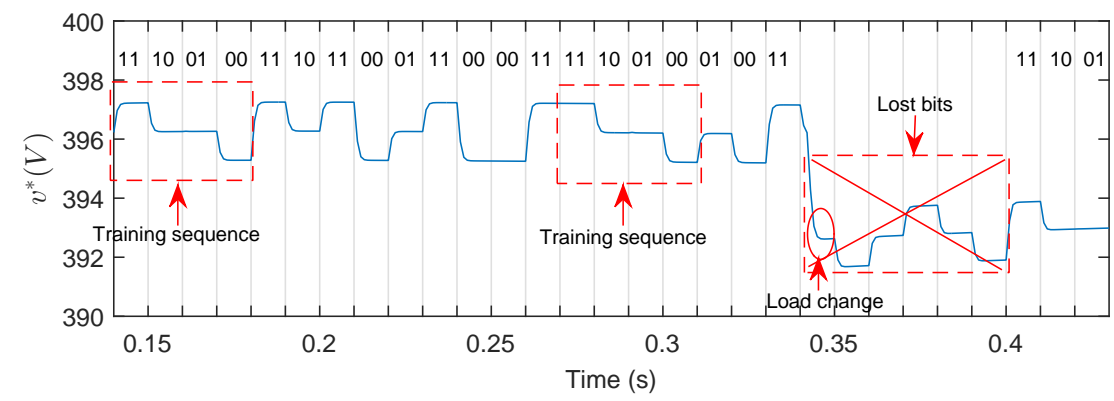

Fig. 13. Power talk with periodic training. $T_{s}=1 \mathrm{~ms}$.

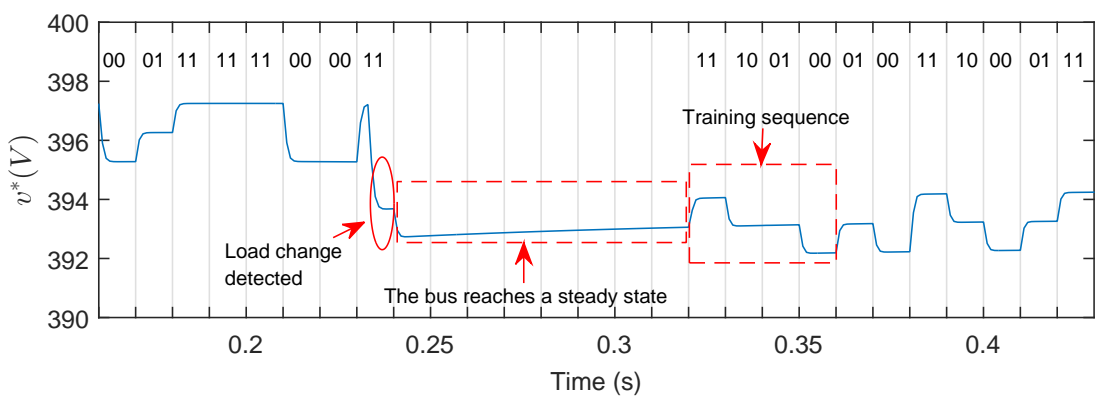

Fig. 14. Power talk with with load change detection. $T_{s}=1 \mathrm{~ms}$.

transmissions with other units. Evidently, the FD binary power talk requires $K$ times more slots than the TDMA version 3

We now turn to potential schemes for activation of the training phase. A simple solution is to perform training periodically and update the detection spaces. A more involved approach would be to employ a model change detector that tracks the bus voltage and, if a change is detected, initiates the training phase. In further text, we investigate and compare the above two schemes. For this purpose, we model the load changes through a Poisson process whose intensity $\lambda$ is the expected number of load changes per time slot. As the slot duration $T_{s}$ is of the order of milliseconds, one can expect that $\lambda<<1$ in practice.

Power Talk with periodic training phase: We assume that the training phase occurs periodically after each VSC transmits $B$ bits of information. Also, we assume the worst-case scenario in

${ }^{3}$ For homogenous and close to ideal systems, all units can construct their detection spaces simultaneously, making the training phase significantly shorter. It can be shown that the minimum number of slots necessary to build all detection spaces in an ideal single bus MG, assuming $M$ slots per point, is $4 M$ for TDMA and $4 K M$ for FD power talk. 
which each system state change completely "destroys" all detection spaces and all following transmissions are lost, see Fig. 13 .

In Appendix $\mathrm{A}-\mathrm{A}$ it is shown that the net transmission rate for TDMA power talk is:

$$
\eta_{\mathrm{TDMA}}=\frac{(1-p)^{L_{\mathrm{TDMA}}+1}\left[1-(1-p)^{K B}\right]}{p\left(L_{\mathrm{TDMA}}+K B\right)} \eta_{\mathrm{TDMA}}^{\mathrm{S}}
$$

where $p=1-e^{-\lambda}$ is the probability that the load changes during a slot and $\eta_{\text {TDMA }}^{\mathrm{S}}$ is the corresponding stable rate, see (43). For FD variant, the net transmission rate is:

$$
\eta_{\mathrm{FD}}=\frac{(1-p)^{L_{\mathrm{FD}}+\frac{1}{\eta_{\mathrm{FD}}^{S}}}\left[1-(1-p)^{\frac{B}{\eta_{\mathrm{FD}}^{S}}}\right]}{\left[1-(1-p)^{\frac{1}{\eta_{\mathrm{FD}}^{S}}}\right]\left(L_{\mathrm{FD}}+\frac{B}{\eta_{\mathrm{FD}}^{\mathrm{S}}}\right)},
$$

where $\eta_{\mathrm{FD}}^{\mathrm{S}}$ is the stable transmission rate, see Table $\amalg$. When $p \rightarrow 0, \eta \rightarrow \frac{B}{L \eta^{\mathrm{S}}+B} \eta^{\mathrm{S}}$, both for TDMA and FD variants. Also, from (45) and (46), one can find the optimal length of the bit block $B$ for given $\lambda$ and $K$, where $B_{\text {opt }}=\max _{B} \eta(B ; K, p)$.

Power Talk with load change tracker: Each VSC can, in principle, locally track $v^{*}$ and $i_{k}$ and decide whether the system state has changed; the state changes should be detected by all units simultaneously with high reliability. An option is to use a standard model change detector [25] that tracks the voltage level. Assume that each VSC implements such state change detector, operated it in the following way: i) if a change is detected, then the current transmission is stopped, ii) $L_{\mathrm{BS}}$ "blank slots" (e.g., nominal operation symbols $\mathbf{x}_{k}^{\mathrm{n}}$ ) are inserted by all units, to allow the system to reach steady state after the load change and, iii) the training phase is re-initiated; as illustrated in Fig. 14. Appendix B derives the following expressions for net transmission rate:

$$
\eta=\frac{1}{p+(1-p)^{-\left(L+L_{\mathrm{BS}}\right)}} \eta^{\mathrm{S}}
$$

which holds both for TDMA and FD power talk. When $p \rightarrow 0, \eta \rightarrow \eta^{S}$.

\section{PERformance EVAluation}

The performance evaluation is obtained using the MG system described in Section II. The bits are equiprobable, i.e., $p_{b}=0.5$ and we use $\gamma=0.1$ to design the input symbol constellation; the effects of the observation noise in this case are virtually negligible, see Section $\mathrm{V}$. 


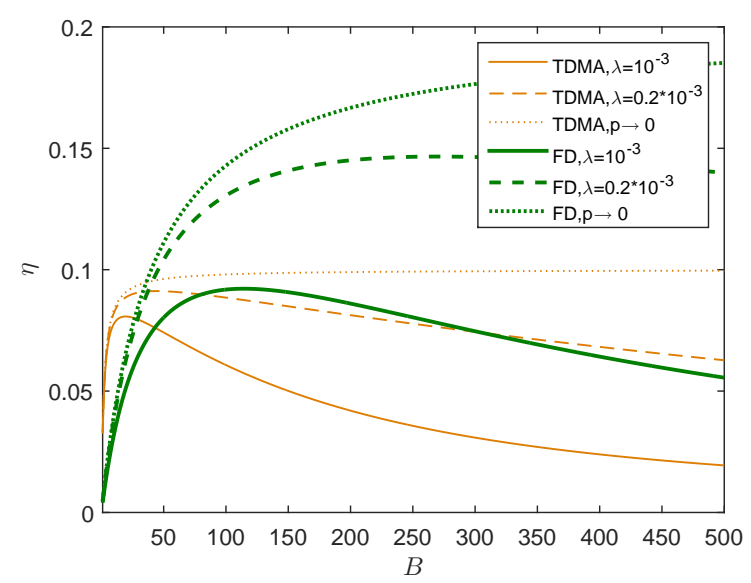

(a) $K=10$.

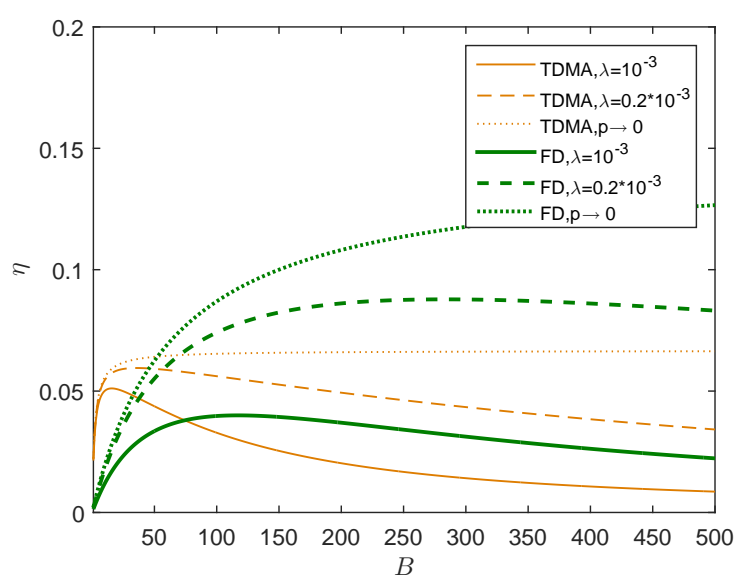

(b) $K=15$.

Fig. 15. $\eta$ as a function of $B, K$ and $\lambda$ : periodic training sequence insertion.

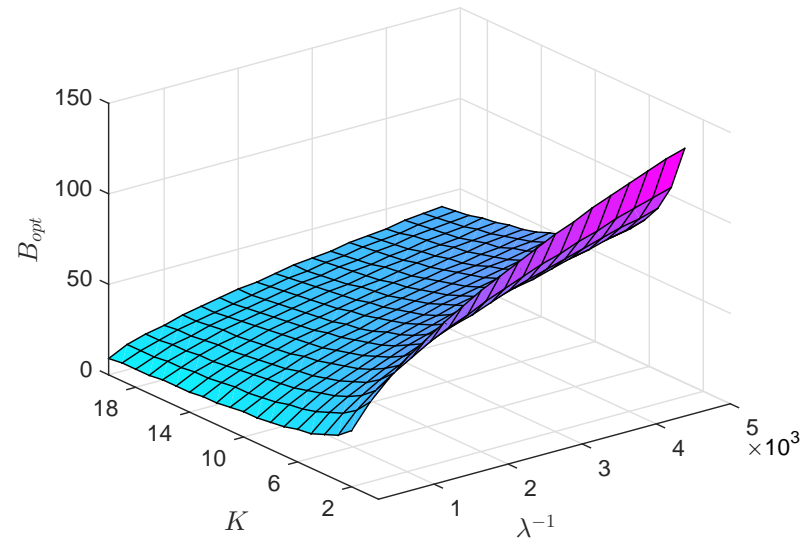

(a) TDMA power talk.

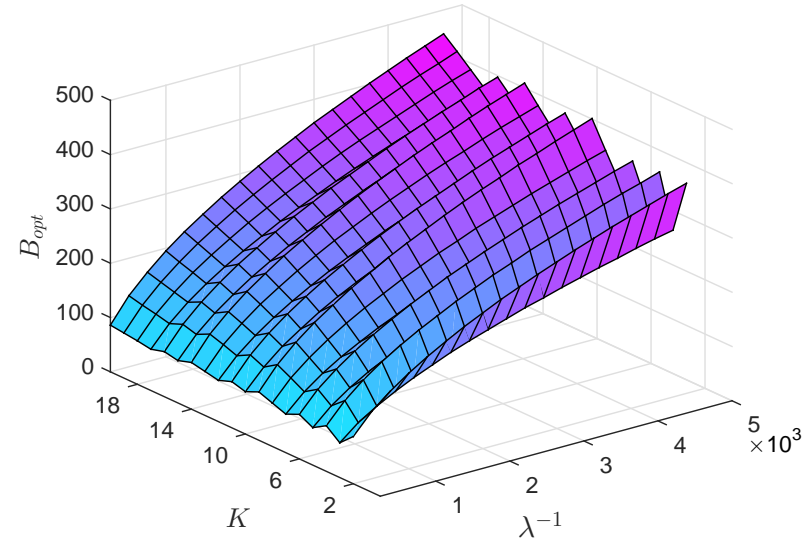

(b) FD power talk.

Fig. 16. The optimal choice of $B$.

\section{A. Dealing with load variations}

Fig. 15 illustrates the net transmission rate per unit $\eta$ for power talk with periodic training phase, as function of $B$ which is the number of transmitted bits after the training phase is reinitiated. In general, for small $B$, the TDMA power talk outperforms the FD variant, and this is more pronounced when the number of units $K$ in the system increases 4

${ }^{4}$ Note that, although the shown net transmission rates seem small, one should bear in mind that in the system with fair scheduling, a maximum transmission rate of $1 / K$ can be achieved using TDMA approach, as illustrated in the figure. 


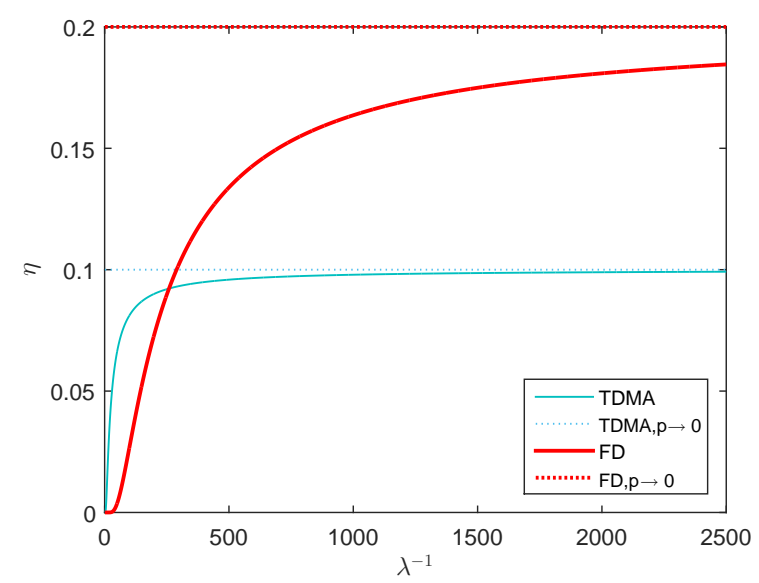

(a) $K=10$.

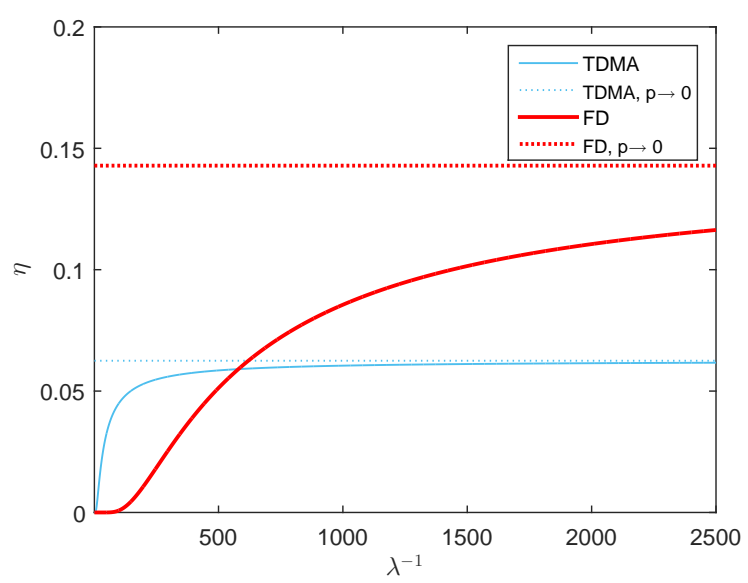

(b) $K=15$.

Fig. 17. $\bar{\eta}$ as a function of $\lambda$ : power talk with load change tracker.

The optimal choice of $B$ that maximizes $\eta$, as function of $K$ and $\lambda$, is depicted in Fig. 16, We note that the depicted values of $B$ are obtained using numerical evaluation; also, the fluctuations of $B_{\text {opt }}$ in case of FD variant are due to the behavior of the optimal coding rate which is a step function of $K$, see Table $\amalg$. Clearly, FD power talk implementation requires longer $B$ to maximize the rate, as it needs to compensate for the overhead of the training phase, i.e., $L_{\mathrm{FD}}>L_{\mathrm{TDMA}}$. Thus, FD power talk is more suitable for longer data sequences when periodic training phase is used.

Fig. 17illustrates the net transmission rate per unit $\eta$ for power talk with load change tracker as function of $\lambda$. Evidently, the FD power talk outperforms the TDMA variant for load change intensities of practical interest, i.e., small $\lambda$. However, TDMA power talk achieves the asymptotic performance faster, which is also a consequence of the length of the training phases.

Fig. 18 compares FD and TDMA power talks in terms of the achievable net transmission rates per unit, respectively, where the results in Fig. 18[(a) are obtained for corresponding $B_{\text {opt }}$. Evidently, $\eta$ decreases monotonically with $K$, which could be expected. Also, FD power talk is clearly a superior solution. As depicted, power talk with model change detector performs significantly better than periodic training strategy for both TDMA- and FD-based solution; the price to pay is increased implementation complexity and sensitivity to potential missed detections and false alarms, which are not included in the present study. Further, for both protocols, the performance loss w.r.t. the stable operation, when $p \rightarrow 0$, is larger for FD-based solution due 


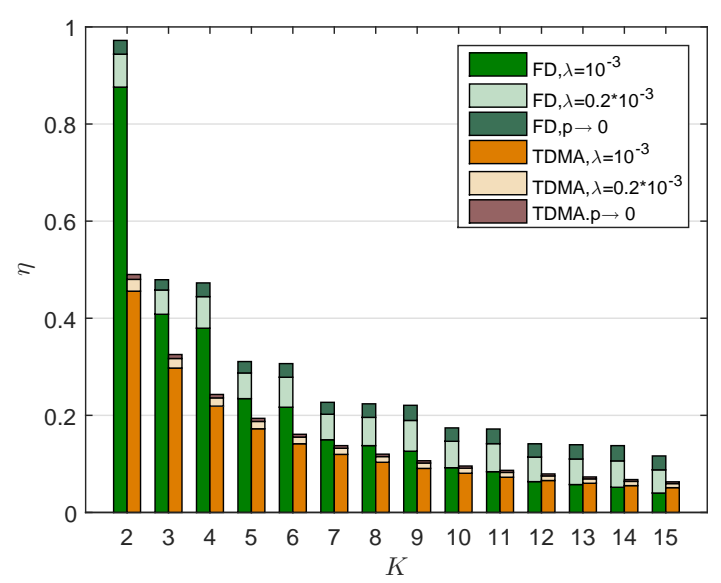

(a) Periodic training phase.

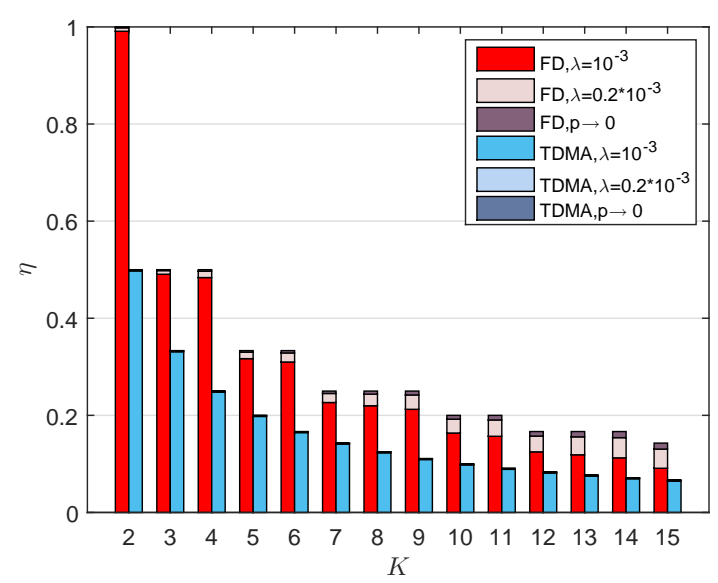

(b) Load change tracker.

Fig. 18. FD vs TDMA power talk for increasing $K$ : net transmission rate per unit $\eta$.

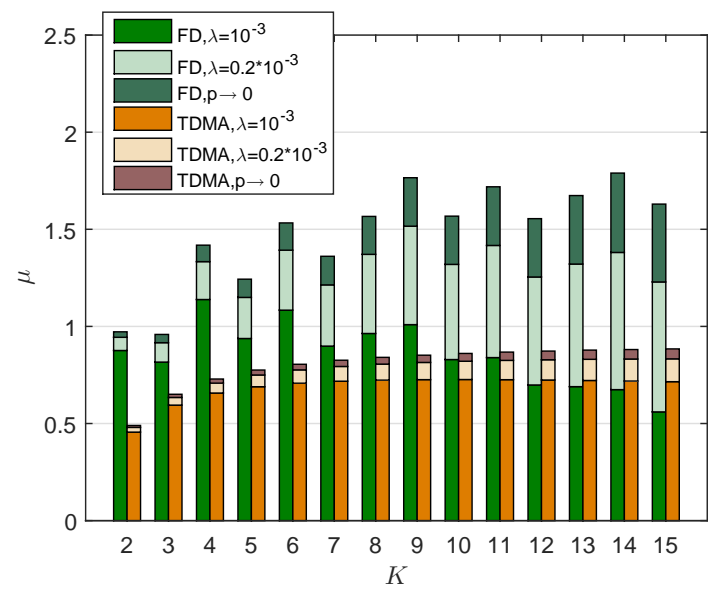

(a) Periodic training phase.

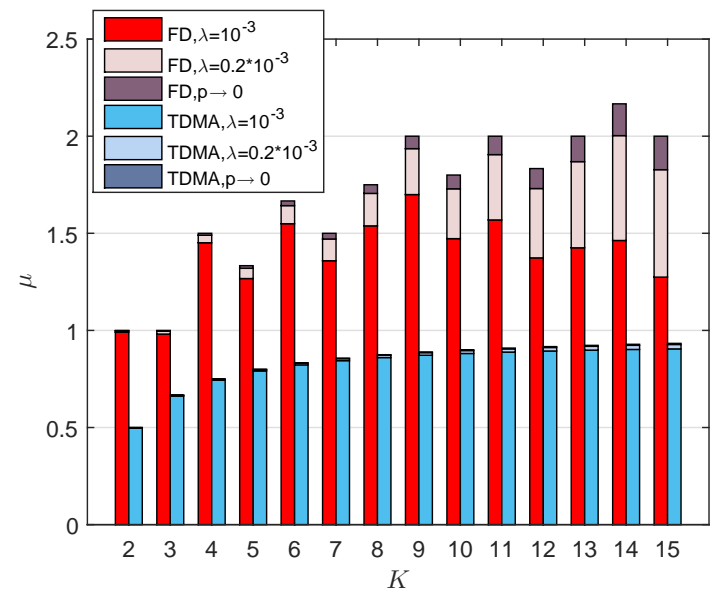

(b) Load change tracker.

Fig. 19. FD vs TDMA power talk for increasing $K$ : net reception rate per unit $\mu$.

to the longer training phase. This deviation becomes more apparent as the number of units $K$ increases, as well as for larger values of the load change intensity $\lambda$. It can be also observed that the gain of the FD-based solution over the TDMA-based solution reduces with increasing $K$ and increasing $\lambda$. In principle, from the equations (45)-(47), one can determine the values of $K$ for which $\eta_{T D M A}>\eta_{F D}$, for given $\lambda$ and $B$. Thus, it could be concluded that FD-based power talk is more efficient for systems with smaller number of units.

We end the evaluation by reviewing the developed power talk protocols in the context of 
applications in which of a particular importance is the amount of information a single unit can obtain about the status of other units in the system. Examples of such applications can be found in distributed control in MGs, average consensus and optimal dispatch. An appropriate metric to evaluate power talk in these applications is the net reception rate, defined as the average number of bits observed by a single unit per slot, denoted with $\mu$ and calculated as $\mu=(K-1) \eta$ when the net transmission rates are equal. Fig. 19 depicts $\mu$ for both protocol implementations. Note that for TDMA binary power talk the asymptotic upper bound for the net reception rate is 1 bit per unit per slot. For FD variant, $\mu$ is significantly larger and increases with $K$ (although not monotonically, due to the coding rates, see Table II). Thus, FD-based power talk can provide significant benefits in MG applications in which information about the status of the rest of the system is necessary.

\section{CONCLUSIONS}

In this paper we presented power talk, a novel concept tailored for communication among units in a MicroGrid. The core idea of power talk is to modulate information using primary control loops of the voltage source converters that regulate the bus voltage. We have shown that it is possible to design signaling constellations that conform to the operating constraints and limits to the power deviations with respect to the nominal operation. We have also shown that using MAP detector at the receiving side practically achieves errorless communication when the load (i.e., power demand) is stable, under mild constraints on the number of units in the system and allowable power deviations.

The main challenge of power talk is the arbitrary variations of load, leading to the uncontrollable and unforeseeable changes of the bus voltage. We investigated techniques to counter-effect load changes, showing that it is possible to optimize the power talk operation given the statistics of the load changes.

The achievable rates of power talk depend on the bandwidth of the primary control loops. In practice, it could be expected that power talk can achieve rates of the order of $100 \mathrm{Baud}-1 \mathrm{kBaud}$. Nevertheless, considering that the inter-MG communications are machine-type in nature, these modest rates may prove to be satisfactory. Moreover, when assessing the potential of power talk, one should also take into account the inherent advantages of power talk, which are use of existing MG power equipment, software implementation, and reliability and availability equal to the reliability and availability of the MG itself. 


\section{APPENDIX A}

\section{ANALYSIS OF POWER TALK WITH PERIODIC INSERTION OF TRAINING SEQUENCES}

\section{A. TDMA power talk}

When scheduled to transmit, VSC $k$ transmits exactly 1 bit of information in a single slot with probability $1-p$, where $p$ is the probability that the load changes during the slot, see Section VI. Under fair scheduling and in the absence of noise, the transmission rates are equal for all units. Denote with $t, 1 \leq t \leq K B$ the slot when the first change of the system state occurs. Then, $\eta_{\mathrm{FD}}$ can be written as:

$$
\eta_{\mathrm{TDMA}}=\frac{(1-p)^{L_{\mathrm{TDMA}}}}{L_{\mathrm{TDMA}}+K B}\left[\frac{p}{K} \sum_{t=1}^{K B}(t-1)(1-p)^{t-1}+B(1-p)^{K B}\right] .
$$

The last term corresponds to the case when no load change occurs during the data phase. Using arithmetic-geometric progression to solve (48) produces (45).

\section{B. FD power talk}

With FD, we use Chang-Weldon uniquely decodable coding and decoding [24]. The code for VSC $k$ contains only two codewords of length $\frac{1}{\eta_{\mathrm{FD}}^{\mathrm{S}}}$ to represent each bit, with $\eta_{\mathrm{FD}}^{\mathrm{S}}$ given in Table II. Thus, when using FD solution, each unit has to send a block of bits of length $\frac{1}{\eta_{\mathrm{FD}}^{\mathrm{s}}}$ correctly to be deliver 1 bit of information that can be uniquely decoded by other units. Again, in absence of noise the transmission rates are equal for all units. Denote with $\tau, 1 \leq \tau \leq B$ the block of bits of length $\frac{1}{\eta_{\mathrm{FD}}^{\mathrm{s}}}$ when the first load change occurs. Then, $\eta_{\mathrm{FD}}$ can be written as:

$$
\eta_{\mathrm{FD}}=\frac{(1-p)^{L_{\mathrm{FD}}}}{L_{\mathrm{FD}}+\frac{B}{\eta_{\mathrm{FD}}^{\mathrm{S}}}}\left[\sum_{\tau=1}^{B}(\tau-1)(1-p)^{\frac{\tau-1}{\eta_{\mathrm{FD}}^{S}}}\left(1-(1-p)^{\frac{1}{\eta_{\mathrm{FD}}^{S}}}\right)+B(1-p)^{\frac{B}{\eta_{\mathrm{FD}}^{S}}}\right] .
$$

Using arithmetic-geometric progression to solve (49), produces (46).

\section{APPENDIX B}

\section{ANALYSIS OF POWER TALK WITH LOAD CHANGE TRACKER}

The derivation is identical for both TDMA- and FD-based power talk. The average number of slots, necessary to deliver $B$ bits of information depends on the number of state changes that occur during the transmission of $B$ bits. In absence of noise, the average number of slots, necessary to deliver $B$ bits is the same for all units and $\eta$ can be written as:

$$
\eta=\frac{B}{\frac{B}{\eta^{S}}+p \frac{B}{\eta^{S}}\left(\mathbb{E}\left\{L^{\prime}\right\}+1\right)}=\frac{1}{1+p\left(\mathbb{E}\left\{L^{\prime}\right\}+1\right)} \eta^{\mathrm{S}},
$$


and it does not depend on $B$ and $\mathbb{E}\left\{L^{\prime}\right\}$ is the average duration of the training phase, since the state can also change during the training. We also include the slot in which the state changed, since the bit in that slot will be retransmitted. To analyze $\mathbb{E}\left\{L^{\prime}\right\}$ we note that if a load change occurs in the training sequence, the training sequence is re initiated. Then, we use the absorbing Markov chain shown on Fig. 20 to model $L^{\prime}$. The chain always starts from the state $L+L_{B S}$.

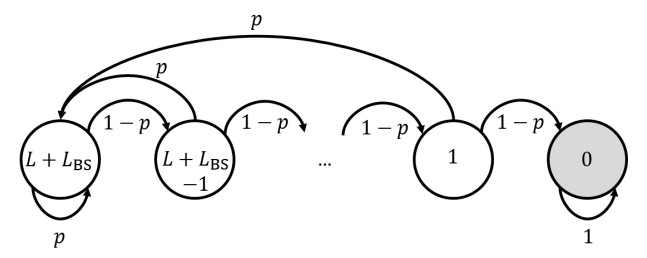

Fig. 20. Absorbing Markov chain as a model for $L^{\prime}$

The absorbing state is the state when the training sequence has been completely sent without interruptions. $\mathbb{E}\left\{L^{\prime}\right\}$ is actually the average number of transitions in the chain until absorption. For the chain shown of Fig. 20, it can be shown that the average number of transitions until absorption is:

$$
\mathbb{E}\left\{L^{\prime}\right\}=\sum_{l=1}^{L+L_{B S}}(1-p)^{-l}
$$

Replacing (51) in (50) and solving the geometric sum, produces (47).

\section{REFERENCES}

[1] R. Lasseter, "Microgrids," in Power Engineering Society Winter Meeting, 2002. IEEE, vol. 1, 2002, pp. $305-308$.

[2] N. H. (Editor), Microgrids: Architectures and Control. Wiley-IEEE Press, 2014.

[3] S. F. Bush, Smart Grid: Communication-Enabled Intelligence for the Electric Power Grid. Elsevier Inc., 2014.

[4] Y. Yan, Y. Qian, H. Sharif, and D. Tipper, “A survey on smart grid communication infrastructures: Motivations, requirements and challenges," IEEE Commun. Surveys Tuts., vol. 15, no. 1, pp. 5-20, First 2013.

[5] J. Guerrero, J. Vasquez, J. Matas, L. de Vicuna, and M. Castilla, "Hierarchical control of droop-controlled ac and dc microgrids; a general approach toward standardization,” IEEE Trans. Ind. Electron., vol. 58, no. 1, pp. 158-172, Jan. 2011.

[6] J. Guerrero, M. Chandorkar, T. Lee, and P. Loh, "Advanced control architectures for intelligent microgrids; part i: Decentralized and hierarchical control," IEEE Trans. Ind. Electron., vol. 60, no. 4, pp. 1254-1262, Apr. 2013.

[7] C. Jin, P. Wang, J. Xiao, Y. Tang, and F. H. Choo, "Implementation of hierarchical control in dc microgrids," IEEE Trans. Ind. Electron., vol. 61, no. 8, pp. 4032-4042, Aug. 2014.

[8] J. Schonberger, R. Duke, and S. Round, "Dc-bus signaling: A distributed control strategy for a hybrid renewable nanogrid," IEEE Trans. Ind. Electron., vol. 53, no. 5, pp. 1453-1460, Oct. 2006.

[9] D. Chen, L. Xu, and L. Yao, "Dc voltage variation based autonomous control of dc microgrids," IEEE Trans. Power Del., vol. 28, no. 2, pp. 637-648, Apr. 2013. 
[10] T. Vandoorn, B. Renders, L. Degroote, B. Meersman, and L. Vandevelde, "Active load control in islanded microgrids based on the grid voltage," IEEE Trans. Smart Grid, vol. 2, no. 1, pp. 139-151, Mar. 2011.

[11] K. Sun, L. Zhang, Y. Xing, and J. Guerrero, "A distributed control strategy based on dc bus signaling for modular photovoltaic generation systems with battery energy storage,” IEEE Trans. Power Electron., vol. 26, no. 10, pp. 3032-3045, Oct. 2011.

[12] S. Galli, A. Scaglione, and Z. Wang, "For the grid and through the grid: The role of power line communications in the smart grid," Proc. IEEE, vol. 99, no. 6, pp. 998-1027, Jun. 2011.

[13] M. Angjelichinoski, C. Stefanovic, P. Popovski, H. Liu, P. Loh, and F. Blaabjerg, "Power talk: How to modulate data over a dc micro grid bus using power electronics," IEEE GLOBECOM 2015, accepted, available at http://arxiv.org/pdf/1504.03016.pdf

[14] M. Angjelichinoski, C. Stefanovic, P. Popovski, and F. Blaabjerg, "Power talk in dc micro grids: Constellation design and error probability performance," IEEE SmartGridComm 2015, submitted, available at http://arxiv.org/pdf/1507.02598.pdf

[15] N. Pogaku, M. Prodanovic, and T. Green, "Modeling, analysis and testing of autonomous operation of an inverter-based microgrid," IEEE Trans. Power Electron., vol. 22, no. 2, pp. 613-625, Mar. 2007.

[16] Y.-R. Mohamed and E. El-Saadany, "Adaptive decentralized droop controller to preserve power sharing stability of paralleled inverters in distributed generation microgrids," IEEE Trans. Power Electron., vol. 23, no. 6, pp. 2806-2816, Nov. 2008.

[17] F. Blaabjerg, Z. Chen, and S. Kjaer, "Power electronics as efficient interface in dispersed power generation systems," IEEE Trans. Power Electron., vol. 19, no. 5, pp. 1184-1194, Sep. 2004.

[18] T. Dragicevic, J. Guerrero, J. Vasquez, and D. Skrlec, "Supervisory control of an adaptive-droop regulated dc microgrid with battery management capability," IEEE Trans. Power Electron., vol. 29, no. 2, pp. 695-706, Feb. 2014.

[19] P. Midya and P. Krein, "Noise properties of pulse-width modulated power converters: open-loop effects," IEEE Trans. Power Electron., vol. 15, no. 6, pp. 1134-1143, Nov. 2000.

[20] S. Mazumder, A. Nayfeh, and D. Boroyevich, "Theoretical and experimental investigation of the fast- and slow-scale instabilities of a dc-dc converter," IEEE Trans. Power Electron., vol. 16, no. 2, pp. 201-216, Mar. 2001.

[21] A. Cavallini, G. Montanari, and M. Cacciari, "Stochastic evaluation of harmonics at network buses," IEEE Trans. Power Del., vol. 10, no. 3, pp. 1606-1613, Jul. 1995.

[22] A. Sangswang and C. Nwankpa, "Random noise in switching dc-dc converter: verification and analysis," in Proc. of IEEE ISCAS '03, Bangkok, Thailand, May 2003.

[23] —_ "Effects of switching-time uncertainties on pulsewidth-modulated power converters: modeling and analysis," IEEE Trans. Circuits Syst. I, Fundam. Theory Appl., vol. 50, no. 8, pp. 1006-1012, Aug. 2003.

[24] S.-C. Chang, "Further results on coding for t-user multiple-access channels (corresp.)," IEEE Trans. Info. Theory, vol. 30, no. 2, pp. 411-415, Mar. 1984.

[25] S. Kay, Fundamentals of Statistical Signal Processing: Estimation Theory, ser. Fundamentals of Statistical Signal Processing. Prentice-Hall PTR, 1998. 